\title{
Der deutsche Beitrag zur internationalen immobilienwirtschaftlichen Forschung
}

\section{Eine empirische Analyse auf Basis von Konferenz- Forschungsbeiträgen}

\author{
Andreas Pfnür · Annette Kämpf-Dern
}

Online publiziert: 26. August 2015

(C) Die Autor(en) 2015. Dieser Artikel ist auf Springerlink.com mit Open Access verfügbar.

Zusammenfassung Der vorliegende Beitrag gibt eine Übersicht über die bisherigen Inhalte immobilienwirtschaftlicher Forschung in Deutschland. Der Beitrag zeigt sowohl die Schwerpunkte als auch die weißen Flecken dieser Forschung im Inland und im internationalen Vergleich auf. Mit Hilfe des Konzepts der Familienähnlichkeiten und Kommensurabilität von Verhaltensannahmen gleichartiger Akteure wurden Cluster immobilienwirtschaftlicher Forschung gebildet. In einer empirischen Studie wurden 14.124 Forschungsbeiträge internationaler Konferenzen der IRES und AREUEA katalogisiert und den Forschungsclustern zugeordnet, deren deutscher Anteil im Fokus dieses Beitrags steht. Es zeigt sich, dass immobilienwirtschaftliche Forschung vor allem aus der Investorenperspektive betrieben wurde, wohingegen Themen aus Sicht der Immobiliennutzung und der Immobilienproduktion/-dienstleistungen weit weniger häufig Gegenstand der deutschen Forschung waren. Die Verteilung der Konferenzbeiträge auf die Cluster immobilienwirtschaftlicher Forschung in Deutschland ist eng korreliert mit der weltweiten Verteilung. Die Bewertung der praktischen Relevanz oder der konzeptionellen Qualität der Beiträge kann und soll durch die Untersuchung nicht vorgenommen werden. Jedoch können anhand der Ergebnisse individuelle Forschungsagenden validiert und gezielt z. B. in Richtung Mainstream oder Nische weiterentwickelt werden. Der Beitrag liefert somit einen innovativen Vorschlag zur Clusterung immobilienwirtschaftli-

\footnotetext{
A. Pfnür $(\square)$

Fachgebiet Immobilienwirtschaft und Baubetriebswirtschaftslehre,

TU Darmstadt,

Darmstadt, Deutschland

E-Mail: pfnuer@bwl.tu-darmstadt.de

A. Kämpf-Dern

Professur für Projektentwicklung und Immobilienmanagement,

HafenCity Universität Hamburg,

Hamburg, Deutschland

E-Mail: annette.kaempf-dern@hcu-hamburg.de
} 
cher Forschung und stellt zum ersten Mal ein umfassendes empirisches Bild über deren inhaltliche Ausrichtung in Deutschland im weltweiten Vergleich dar.

Schlüsselwörter Immobilienwirtschaftliche Forschung ·

Deutscher Anteil internationaler Forschung · Forschungsstrategien ·

Forschungsthemen · Forschungshistorie

Abstract This article seeks to identify the past activity and interest factors that drove German real estate research, as well as the correlation of these factors with international research activities. The aim is to provide a present view of Germany's strong and weak areas of real estate research compared to the international situation.

The findings of this paper are critical in providing a knowledge base of the status quo in real estate research. This overview can be used as a map for researchers to identify their positions in the community of real estate research and in consequence to focus on future research activities. Given that real estate research has expanded into a world-wide network, it is necessary to consider the international links of these findings. In this article, the analysis focuses on identifying the key areas as well as white spots of future research fields, but will not evaluate the respective research activities.

Design: Data was taken from international real estate conferences as these bodies had the most diversified and relevant data both in term of quality and quantity. To ensure relevance to the study, the papers had to meet three criteria. They had to:

a. address real estate or a real estate related services;

b. analyze and discuss issues of economics, business economics or management; and

c. apply a scientific approach in their study.

In order to analyze the massive amount of data in the form of articles, cluster characteristics were identified and assigned to the data. For this, a "3-perspectives model" of real estate actors and activities was used. This model explains the goals, functions and claims of real estate for the user, producer, and investor. Research fields were defined through using this model, and within the perspectives differentiated by real estate type and activities.

All data for the study was taken from conference papers of ARES, AREUEA, AsRES, ERES, LARES, MENARES and PRRES conferences between 1993 and 2012. This provided a total of 14.124 samples from 136 conferences held. The final analysis was then conducted with the grounded theory approach.

Findings: Lead authors with German origins contributed to $3.8 \%$, or 533 papers in total. These were primarily found in ERES, ARES, and PRRES conferences. Overall, German participation in international research regarding real estate ranks fifth, after the United States, Australia, China, and UK. In the last two decades, the German share has risen from around 1\% in 1993 to more than 5\% in 2012. A similar growth rate was found in ERES conferences, where the German share grew from $5 \%$ to nearly $15 \%$.

In German papers, overall, the focus on the investor perspective was twice as big as that of the production or user perspective. Interestingly, this distribution shows a high correlation with international participation. 
In the more detailed and diverse subcategories, the priorities between German and international research were found to be much more heterogeneous. While the international focus was more on market cycles, housing-related topics, urban development, or brokerage, German research tended to focus on education, corporate real estate management, and property development. The 0.66-quantiles show that German real estate research has a higher subcategory density compared to international research: Two thirds of German research is covered by 26 different subcategories whereas the same is covered by 34 subcategories on an international level. A moderate statistical dependence of German research on international trends was found.

It is worth noting that the topics of German research - compared to global activities - are in less-developed fields such as portfolio management, education, securities, investment, and IPO-related topics. Analysis from this study also shows that German research topics to a high degree affect topics that are internationally ranked top 10. According to the impact factor found, German research is especially important for topics like education, REITs, valuation, retail/office/logistics real estate markets, and investment topics. Additionally, an analysis of German research institutions show that universities are driving real estate research, with more than $70 \%$ of all research done at universities.

The findings of the study show that German real estate research -quantitatively analyzed - is well aligned with international priorities, and accordingly impacts and contributes to the body of knowledge. As to whether the outcome and subsequent research strategies are significantly relevant on a qualitative level will have to be further examined in future studies.

Keywords Real Estate Research - German share of research · Research Strategies · Research Topics · Research History

\section{Problemstellung}

An deutschen Hochschulen gibt es mittlerweile eine in den letzten Jahren stark gewachsene Anzahl an Arbeitsbereichen, Fachgebieten und Lehrstühlen, an denen maßgeblich immobilienwirtschaftliche Forschung betrieben wird. Von diesen Lehrstühlen ist zudem ein signifikant hoher Anteil an Universitäten angesiedelt. Diese haben im Vergleich zu Fachhochschulen und Berufsakademien einerseits einen naturgemäß stärkeren Forschungsbezug und verfügen zudem über das Promotionsrecht. Deshalb sollten sie sich in der wissenschaftlichen Forschung in besonderem Maße einbringen. ${ }^{1}$ Jeder Lehrstuhl legt im Rahmen seiner eigenen Forschungsstrategie die inhaltliche Ausrichtung seiner Forschungsarbeiten fest und entwickelt diese sukzessive fort. Hierfür, genauso wie für die Definition der eigenen Positionierung,

\footnotetext{
${ }^{1}$ Aufgrund von Abgrenzungsproblemen ist die Nennung einer exakten Zahl der immobilienwirtschaftlichen Lehrstühle/Organisationseinheiten ohne eine umfangreiche Befragung nicht möglich. So wird ohne eigene Untersuchung kaum zu klären sein, ob einzelnen Einheiten nach Art und Umfang hinreichend intensiv immobilienwirtschaftliche Forschung betreiben, um hier gezählt werden zu können. Einen ersten Anhaltspunkt gibt die Adressliste der Gesellschaft für immobilienwirtschaftliche Forschung e. V. aus dem August 2014. Sie zählt 191 Einträge an immobilienwirtschaftlich arbeitenden Organisationseinheiten.
} 
ist eine regelmäßige Bestandsaufnahme der bisher geleisteten Arbeiten notwendig. Allerdings reicht es aufgrund der zunehmenden internationalen Vernetzung der Forschungslandschaft nicht mehr aus, sich nur national zu orientieren. Vielmehr sind die Berücksichtigung der weltweiten Forschungstätigkeiten und damit eine internationale Einbettung notwendig.

In diesem Beitrag wird deshalb dargestellt, auf welchen Gebieten und zu welchen Problemen in der Vergangenheit in der deutschen immobilienwirtschaftlichen Forschung gearbeitet wurde und welchen Anteil die deutschen Kolleginnen und Kollegen in welchen Themenfeldern an der jeweiligen weltweiten Forschung haben. Daraus werden Rückschlüsse auf die spezifischen Schwerpunkte deutscher immobilienwirtschaftlicher Forschung gezogen und weiße Flecken sowie Schwachstellen identifiziert.

Das Forschungsziel besteht somit darin, der deutschen immobilienwirtschaftlichen Forschungsgemeinschaft durch einen allgemeingültigen Bezugsrahmen und dessen bisherige Ausfüllung im internationalen Vergleich Anhaltspunkte für die Entwicklung, Fortschreibung bzw. Veränderung von Forschungsstrategien zu liefern. Hierdurch können Schwerpunkte und Nischen bisheriger Forschung identifiziert werden und die Einordnung der eigenen Tätigkeiten erfolgen. Darüber hinaus können die hier erarbeiteten Ergebnisse als Maßstab zur kritischen Reflektion und als Impulse zur Weiterentwicklung der inhaltlichen Ausrichtung spezifischer Forschungsagenden von Akteuren und Institutionen immobilienwirtschaftlicher Forschung herangezogen werden. Explizit kein Ziel dieses Beitrags ist es, Aussagen zur Qualität von Beiträgen oder Institutionen oder zu den Chancen und Risiken der zukünftigen Prosperität einzelner Forschungsthemen zu machen.

\section{Umrisse und innere Struktur immobilienwirtschaftlicher Forschung}

\subsection{Was ist immobilienwirtschaftliche Forschung?}

Entsprechend des Ziels dieses Beitrags wird hier von einem möglichst weiten Begriff immobilienwirtschaftlicher Forschung ausgegangen. Ob es sich in diesem Sinne um für die hier vorgenommene Betrachtung relevante Inhalte handelt, wird an drei notwendige und hinreichende Bedingungen geknüpft: ${ }^{2}$

1. Das Erfahrungsobjekt des Beitrags ist die Immobilie und/oder eine Dienstleistung, die zur Zweckbestimmung der Immobilie in ihrem Lebenszyklus unmittelbar beiträgt.

2. Das Erkenntnisobjekt des Beitrags ist zumindest einem der Bereiche Betriebswirtschaftslehre, Volkswirtschaftslehre oder Management zweifelsfrei zuzuordnen. Damit wird insbesondere auch die Forschung auf dem Gebiet der Baubetriebswirtschaftslehre als ein Bestandteil der immobilienwirtschaftlichen Forschung berücksichtigt.

\footnotetext{
${ }^{2}$ Vgl. Kämpf-Dern, A. et al., Perspectives 2013, S. 4.
} 
3. Der Beitrag ist methodisch angelegt und folgt wissenschaftlich anerkannten Standards.

Die hier verwendete Begriffsfassung entspricht dem international üblichen Begriff des „Real Estate Research“. Eine Unterscheidung zwischen ,immobilienwirtschaftlicher Forschung“ und ,immobilienökonomischer Forschung“, wie sie auf Konferenzen in Deutschland anzutreffen ist, wird hier nicht getroffen. ${ }^{3}$ Vielmehr geht es um die Vereinigungsmenge beider Begriffe.

\subsection{Drei Perspektiven Modell der immobilienwirtschaftlichen Forschung}

Neben der Abgrenzung immobilienwirtschaftlicher Forschung nach außen bedarf es für die folgende Analyse des Beitrags deutscher Forscher zur weltweiten immobilienwirtschaftlichen Forschung einer inneren Struktur dieses Forschungsgebiets.

Beim Versuch der Gliederung immobilienwirtschaftlicher Forschung wird schnell klar, dass bisher keine monothetische Taxonomie, die eine eindeutige und bidirektionale baumartige Struktur immobilienwirtschaftlicher Forschungsthemen abbildet, existiert. Vielmehr bestehen zwischen immobilienwirtschaftlichen Forschungsarbeiten systemisch vernetzte Strukturen. Auf den systemischen Zusammenhang immobilienwirtschaftlicher Forschungsbeiträge deuten beispielsweise auch bibliometrische Studien ${ }^{4}$ und semantische Inhaltsanalysen ${ }^{5}$ hin.

Wenn monothetische Taxonomien nicht geeignet sind, stellt sich die Frage nach brauchbaren Alternativen. Die Gesellschaftswissenschaften bedienen sich in vergleichbaren Anwendungen, in denen Objekte inhaltlich überlappender Kategorien (polythetisch) zu systematisieren sind, oft des philosophischen Konzepts der Familienähnlichkeiten von Ludwig Wittgenstein. ${ }^{6}$ Zur Zuordnung von Forschungsobjekten zu immobilienökonomischen Forschungsfamilien sind zunächst auf sehr hoher Ebene aussagefähige Attribute zu identifizieren, die zu möglichst unterschiedlichen Familien mit in sich idealer Weise hohen Ähnlichkeiten führen. In der Immobilienwirtschaft werden gleichartige Fragestellungen regelmäßig nach der Nutzungsart, nach dem Funktionsbereich oder nach der Stufe der Wertschöpfungskette gebildet. Alle unsere Versuche, nach einem dieser Ordnungskriterien die nachfolgend untersuchte enorme Anzahl immobilienwirtschaftlicher Forschungsarbeiten in einer überschaubaren Anzahl an Clustern zu ordnen, schlugen fehl.

In den Gesellschaftswissenschaften werden regelmäßig Forschungscluster anhand von gleichgerichteten Annahmen über das Verhalten von Akteuren gebildet. Auch in der Ökonomie im Allgemeinen und der Immobilienwirtschaft im Besonderen muss diese allgemeingültige Forderung nach der Verhaltenskommensurabilität gleichartiger, d. h. unmittelbar vergleichbarer und modellhaft anschlussfähiger Forschung

\footnotetext{
${ }^{3}$ Vgl. zum Beispiel Schultes Darstellung auf dem 20-jährigen Jubiläum der Gesellschaft für Immobilienwirtschaftliche Forschung, auch dargestellte in der Broschüre: Gif im Fokus: 20 Jahre gif! Schulte (2014) S. 5 f.

${ }^{4}$ Vgl. Hardin III et al., Influential 2006 und Hardin III et al., Citation 2007.

${ }^{5}$ Vgl. Winson-Geideman, K. und Evangelopoulos, N., Topics 2013.

${ }^{6}$ Vgl. Wittgenstein, L., Philosophical Investigations 1953, Teil I, insb. PU 67.
} 
gelten. ${ }^{7}$ Wenn beispielsweise ein Nutzer in seiner Standortentscheidung die Minimierung der Nutzungskosten anstrebt, ein Investor hingegen die Rendite auf sein im gleichen Projekt eingesetztes Kapital zu maximieren gedenkt und der Ersteller eben dieser Immobilie seine Umsatzrendite maximieren wird, ist das Verhalten aller drei Akteure in einer Projektentwicklung höchst unterschiedlich. Forschung, die diese Unterschiede ignoriert, bezeichnet Kuhn als inkommensurabel. Werden in unterschiedlichen modellhaften Abbildungen der Realität gleiche oder zumindest sehr ähnliche Annahmen über das Verhalten der Akteure getroffen, so weisen diese Arbeiten Familienähnlichkeiten im Wittgensteinschen Sinne auf und gehören folglich in ein gemeinsames Cluster.

Immobilien sind als Wirtschaftsgüter und Erfahrungsobjekte einzigartig (und komplex), da sie die folgenden drei verhaltensleitenden Zwecke simultan mit vergleichbarer Intensität und Einfluss auf die Märkte erfüllen: ${ }^{8}$

1. Immobilien dienen als Betriebsmittel/Ressource im Leistungserstellungsprozess

Immobilien gehen als Input in betriebswirtschaftliche Transformationsprozesse ein. Dies gilt insbesondere für die Nutzung von Gewerbeimmobilien, im übertragenen Sinne aber - als Konsumtionsprozeese - auch für Wohnimmobilien. Wirtschaftssubjekte haben hier die Perspektive des Nutzers der Immobilie. Sie verfolgen regelmäßig das Ziel der Maximierung des Verhältnisses von Nutzen und Kosten des Immobilieneinsatzes.

2. Immobilien und immobilienwirtschaftliche Dienstleistungen sind das Ergebnis eines Leistungserstellungsprozesses

Im Lebenszyklus werden während Konzeption, Planung, Bau, Betrieb und Verwertung regelmäßig Leistungen erbracht, die die Immobilie selbst und ihre ökonomische Nutzenstiftung produzieren. Die Wirtschaftssubjekte haben hier die Perspektive des Produzenten. Sie verfolgen durch die Produktion regelmäßig das Ziel der Gewinnerzielung.

\section{Immobilien dienen der Kapitalanlage}

Die hohe Kapitalintensität gepaart mit der Möglichkeit der Einkommens- und Wertzuwachserzielung machen immobiliare Wirtschaftsgüter zu einer Kapitalanlage. Die Wirtschaftssubjekte haben hier die Perspektive des Eigentümers, der sein Kapital anlegt. Sie verfolgen regelmäßig das Ziel der Maximierung des Werts des eingesetzten Kapitals.

Die folgende Abb. 1 fasst die Überlegungen zum 3-Perspektiven-Modell immobilienwirtschaftlicher Forschung graphisch zusammen:9

Wie bei ihrer Beschreibung deutlich wurde, sind die drei Perspektiven untrennbar mit dem Verhalten der Wirtschaftssubjekte in Bezug auf das Wirtschaftsgut Immobilie verbunden. Allerdings ist das Modell als Sichtenmodell zu verstehen, in dem ein Akteur durchaus auch zwei oder alle drei Perspektiven auf sich vereinen kann.

\footnotetext{
${ }^{7}$ Vgl. Kuhn, T. S., Scientific Revolutions 2012, S. 116.

${ }^{8}$ Vgl. Kämpf-Dern, A. und Pfnür, A., Grundkonzept 2009, S. 16 f.

${ }^{9}$ Vgl. Kämpf-Dern u. Pfnür (2009) sowie zur weiteren Erläuterung des Konzepts Pfnür (2011), S. 22 ff..
} 
Abb. 1 3-Perspektiven-Modell der immobilienwirtschaftlichen Forschung

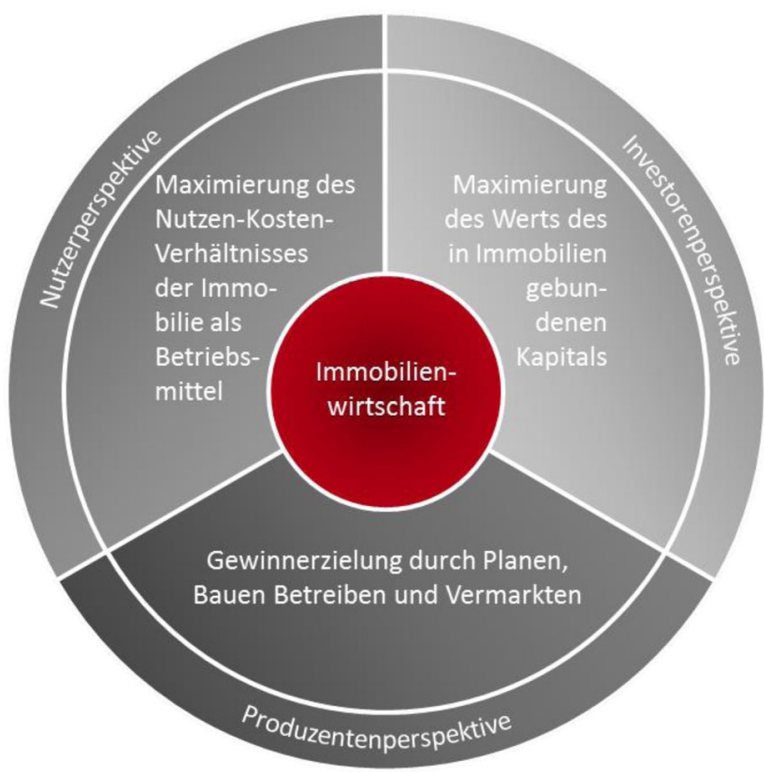

2.3 Bezugsrahmen zur Kodierung und Kategorisierung immobilienwirtschaftlicher Forschung in Deutschland

Die nachfolgende Kodierung und Kategorisierung der immobilienwirtschaftlichen Forschung erfolgt auf Basis des o. g. Perspektiven-Modells als übergeordnetem Cluster-Attribut, nicht auf den sonst häufig verwendeten Lebenszyklusphasen. Da bestimmte Akteursgruppen typischerweise gleichzeitig in Bezug auf zwei oder sogar alle drei Perspektiven agieren (beispielsweise „Eigennutzer“ in der Nutzer- und in der Investorenperspektive, oder Kommunen mit öffentlich finanzierten und betriebenen Immobilien in allen drei Perspektiven), werden im Bezugsrahmen neben den „reinen“ Perspektiven „Nutzer“/,User“, „Produzent“/,Producer“ und „Investor“ auch „Schnittstellen-Perspektiven“ „Investor-User“, „User-Producer“, „InvestorProducer“ und „Investor-User-Producer“ verwendet. Eine zusätzliche, sozusagen überlagernde Kategorie auf Perspektiven-Level ist die des „Regulator“, die die normen- und gesetzgebenden Akteure einer Gesellschaft und deren Ziele repräsentieren. Die Perspektiven stellen das dominierende Attribut bzw. Kriterium der nachfolgenden Kategorisierung immobilienwirtschaftlicher Forschungsbeiträge dar.

Zur vertiefenden Beschreibung und Strukturierung dienen zwei weitere, nachgelagerte Attribute, die damit die Untergruppen der Perspektiven-Cluster beschreiben (siehe Tab. 1).

Das ist zum einen der Immobilientyp („RE Type“). Zum anderen wird nach der Art der BezugsAKTIVITÄT („RE Activity“) nach „RE Management“ versus ,,(Third Party) RE Services“ unterschieden. Zusätzlich - aber nicht weiter differenziert können Forschungsarbeiten der Kategorie „Education“ zugeordnet werden, wenn sie sich mit Aus- und Weiterbildungsthemen der Immobilienwirtschaft beschäftigen. 
Tab. 1 Übersicht immobilienwirtschaftlicher Forschungsfelder. (Quelle: Kämpf-Dern, A. et al., Perspectives 2013, S. 21)

\begin{tabular}{|c|c|c|c|c|c|c|c|c|}
\hline Perspectives & Regulator & Investor & $\begin{array}{l}\text { Investor/ } \\
\text { User }\end{array}$ & User & $\begin{array}{l}\text { User/ } \\
\text { Producer }\end{array}$ & Producer & $\begin{array}{l}\text { Producer/ } \\
\text { Investor }\end{array}$ & $\begin{array}{l}\text { Investor/ } \\
\text { User/ } \\
\text { Producer }\end{array}$ \\
\hline \multicolumn{9}{|l|}{$\begin{array}{l}\text { RE Property/ } \\
\text { Activity type }\end{array}$} \\
\hline \multicolumn{9}{|l|}{$\begin{array}{l}\text { Envionment, } \\
\text { Land, Rural, } \\
\text { Urban }\end{array}$} \\
\hline \multicolumn{9}{|l|}{ Commercial RE } \\
\hline \multicolumn{9}{|l|}{$\begin{array}{l}\text { Residential RE/ } \\
\text { Housing }\end{array}$} \\
\hline \multicolumn{9}{|l|}{$\begin{array}{l}\text { General } \\
\text { Markets }\end{array}$} \\
\hline $\begin{array}{l}\text { Real Estate } \\
\text { Management }\end{array}$ & $\begin{array}{l}\text { e.g. Corpo- } \\
\text { rate Go- } \\
\text { vernance }\end{array}$ & $\begin{array}{l}\text { e.g. } \\
\text { Portfolio } \\
\text { Manage- } \\
\text { ment }\end{array}$ & $\begin{array}{l}\text { e.g. } \\
\text { Center } \\
\text { Manage- } \\
\text { ment }\end{array}$ & $\begin{array}{l}\text { e.g. } \\
\text { CRE } \\
\text { Port- } \\
\text { folio } \\
\text { Ma- } \\
\text { nage- } \\
\text { ment }\end{array}$ & $\begin{array}{l}\text { e.g. } \\
\text { Facility } \\
\text { Manage- } \\
\text { ment }\end{array}$ & $\begin{array}{l}\text { e.g. } \\
\text { Property } \\
\text { Company } \\
\text { Managment }\end{array}$ & $\begin{array}{l}\text { e.g. Asset } \\
\text { Manage- } \\
\text { ment }\end{array}$ & $\begin{array}{l}\text { e.g. } \\
\text { CREM }\end{array}$ \\
\hline $\begin{array}{l}\text { Third Party RE } \\
\text { Services }\end{array}$ & & & & & $\begin{array}{l}\text { e.g. CRE } \\
\text { Services }\end{array}$ & $\begin{array}{l}\text { e.g. } \\
\text { Planning } \\
\text { Brokerage }\end{array}$ & $\begin{array}{l}\text { e.g. } \\
\text { Appraisal }\end{array}$ & \\
\hline
\end{tabular}

Dies ist ein idealtypischer Ansatz, der auf den (aus den vorhandenen Informationen erkennbaren) Fokus eines Forschungsbeitrags abstellt, während in der Realität die Grenzen eben nicht trennscharf sind (polythetischer Ansatz) und in dem einen oder anderen Fall durchaus die konkrete Zuordnung auch zu einem anderen Attribut bzw. einer anderen Attributekombination erfolgen könnte, was jedoch angesichts der Datenmengen und zugunsten der Auswertbarkeit akzeptiert wird.

\section{Empirische Untersuchung}

\subsection{Datensatz und methodologisches Vorgehen}

Als Maßstab des Forschungsbeitrags wurden hier ganz bewusst die internationalen Immobilienkonferenzen gewählt. In den Arbeiten von Roulac et al. sowie WinsonGeideman/-Evangelopoulos wurden demgegenüber wissenschaftliche Publikationen als Maßstab herangezogen. ${ }^{10}$ Eine weitere, von Schulte/Bulwien/Sturm/Zimmermann verfolgte Möglichkeit besteht in der Befragung von Forschern zur Einschätzung der Wichtigkeit alternativer Forschungsthemen. ${ }^{11}$ Johnson et al. sehen den Vorteil der Auswertung von Konferenzbeiträgen gegenüber Publikationen darin begründet, dass eine wesentlich größere Anzahl und Breite an Forschungsthemen untersucht wer-

${ }^{10} \mathrm{Vgl}$. Roulac, S. E. et al., Evolving 2005 sowie Winson-Geideman, K. und Evangelopoulos, N., Topics, 2013.

${ }^{11}$ Vgl. Schulte, K.-W. et al., Forschungsschwerpunkte 2004. 
Tab. 2 Verteilung der Anzahl der ausgewerteten Beiträge auf Konferenzen

\begin{tabular}{|c|c|c|c|c|c|c|c|c|c|c|c|}
\hline \multicolumn{12}{|c|}{ Konferenzen (-: es hat keine Konferenz stattgefunden) } \\
\hline$\overline{\mathrm{Jahr}}$ & AfRES & ARES & $\begin{array}{l}\text { ARE- } \\
\text { UEA } \\
\text { Annual }\end{array}$ & $\begin{array}{l}\text { ARE-UEA } \\
\text { Interna- } \\
\text { tional }\end{array}$ & $\begin{array}{l}\text { ARE- } \\
\text { UEA } \\
\text { Mid-Year }\end{array}$ & AsRES & ERES & LARES & $\begin{array}{l}\text { ME- } \\
\text { NA- } \\
\text { RES }\end{array}$ & PRRES & Sum-me \\
\hline 1993 & - & 143 & 100 & 64 & 21 & - & - & - & - & - & 328 \\
\hline 1994 & - & 173 & 110 & - & 21 & - & 43 & - & - & - & 347 \\
\hline 1995 & - & 172 & 115 & - & 23 & - & 96 & - & - & 1 & 407 \\
\hline 1996 & - & 146 & 118 & 76 & 22 & - & 67 & - & - & 2 & 431 \\
\hline 1997 & - & 137 & 122 & - & 19 & - & 94 & - & - & 2 & 374 \\
\hline 1998 & - & 164 & 117 & - & 19 & - & 105 & - & - & 3 & 408 \\
\hline 1999 & - & 149 & 96 & - & 16 & - & 108 & 22 & - & 62 & 453 \\
\hline 2000 & - & 160 & 97 & - & 19 & - & 81 & - & - & 72 & 429 \\
\hline 2001 & 26 & 106 & 90 & - & 30 & - & 205 & 28 & - & 72 & $\mathbf{5 5 7}$ \\
\hline 2002 & - & 147 & 96 & - & 26 & 114 & 143 & 12 & - & 82 & 620 \\
\hline 2003 & - & 166 & 96 & 67 & 24 & 156 & 215 & - & - & 75 & 799 \\
\hline 2004 & - & 158 & 89 & 67 & 36 & 77 & 223 & 38 & - & 52 & 740 \\
\hline 2005 & - & 162 & 97 & 43 & 29 & 114 & 263 & 56 & - & 90 & 854 \\
\hline 2006 & 56 & 175 & 94 & - & 35 & 176 & 227 & 36 & - & 97 & 896 \\
\hline 2007 & 57 & 226 & 96 & - & 41 & 252 & 309 & 48 & - & 78 & 1107 \\
\hline 2008 & 37 & 167 & 92 & 97 & 38 & 318 & 222 & 42 & - & 88 & 1101 \\
\hline 2009 & 62 & 190 & 90 & - & 61 & 187 & 295 & 59 & - & 133 & 1077 \\
\hline 2010 & 48 & 212 & 91 & 70 & 54 & 169 & 332 & 65 & 10 & 108 & 1159 \\
\hline 2011 & 22 & 219 & 86 & - & 58 & 189 & 230 & 52 & 11 & 112 & 979 \\
\hline 2012 & 63 & 259 & 83 & - & 59 & 177 & 288 & - & - & 129 & 1058 \\
\hline Sum & 371 & 3431 & 1975 & 484 & 651 & 1929 & 3546 & 458 & 21 & 1258 & 14.124 \\
\hline
\end{tabular}

den können. ${ }^{12}$ Zudem geben die Konferenzen einen aktuelleren Forschungsstand wieder, als es langfristige Publikationsprozesse in Journals könnten. Der Nachteil dieses Vorgehens besteht vor allem darin, dass eine Qualitätsgewichtung der untersuchten Konferenzbeiträge entfällt, wie sie auf Basis von beispielsweise Journalrankings zumindest nach dem Kriterium der methodischen Qualität (rigor) ansatzweise möglich wäre. ${ }^{13}$ Weiterhin ist die Teilnahme an wissenschaftlichen Konferenzen im Gegensatz zu Publikationen in wissenschaftlichen Zeitschriften für eine wissenschaftliche Karriere in den Wirtschaftswissenschaften von untergeordneter Bedeutung, so dass Forscher zugunsten einer Konzentration auf ihre Publikationen, mangels ausreichender finanzieller Ressourcen oder aus anderen Gründen auf eine Konferenzteilnahme verzichten könnten.

Um eine möglichst eindeutige Abgrenzung der immobilienwirtschaftlichen Konferenzen vornehmen zu können, beziehen wir alle bislang unter dem Dach der International Real Estate Society sowie der American Real Estate and Urban Economics Association zwischen 1993-2012 durchgeführten Konferenzen als Grundgesamtheit in unsere Untersuchung ein. Insgesamt handelt es sich um mittlerweile neun bzw. zehn Konferenzen pro Jahr, die von den folgenden acht unterschiedlichen Forschungsgesellschaften ausgerichtet werden (siehe Tab. 2):

\footnotetext{
${ }^{12}$ Vgl. Johnson, L. L. et al., ARES Paper 1996.

${ }^{13}$ Vgl. Schrader, U. und Hennig-Thurau, T., VHB-JOURQUAL 2009.
} 
Die AREUEA, die zugleich die älteste der oben genannten Forschungsgesellschaften ist, richtet gleich drei jährlich stattfindende Konferenzen aus. Neben der nationalen AREUEA Assa Conference (AREUEA anual), gibt es ein ,national meeting“ (AREUEA midyear) sowie ein ,international meeting“ (AREUEA international). Insgesamt besteht die Grundgesamtheit der Analyse aus 14.124 Datensätzen, die alle bisher erhältlichen Präsentations-Beiträge (keine Panel-Sitzungen), d. h. ca. 95\% der Beiträge der 136 Konferenzen im Zeitraum von 1993 bis 2012 enthalten (siehe Tab. 2).

Darüber hinaus gibt es eine Vielzahl an weiteren Forschungskonferenzen, die immobilienwirtschaftliche Themenstellungen entweder mitbehandeln oder in engeren, vertieften Ausschnitten zum Gegenstand haben. Eine vollständige Auflistung ist an dieser Stelle weder möglich noch zielführend. Exemplarisch hinzuweisen ist aber auf die jährlich im Herbst stattfindende Konferenz recapnet des Mannheimer ZEW (www.recapnet.org).

Aus den vorgenannten Gründen kann und soll deshalb nicht der Anspruch auf Vollständigkeit erhoben werden. Gleichwohl dürfte die International Real Estate Society und American Real Estate and Urban Economics Association in der Summe im Wesentlichen ihren eigenen Ansprüchen als Dachorganisationen der weltweiten immobilienwirtschaftlichen Forschung recht weitgehend gerecht werden.

Die hier ausgewerteten Informationen wurden über die Webseiten der Gesellschaften sowie durch individuelle Anfragen bei den Gesellschaften in einer zu diesem Zweck angelegten Excel-Datenbank zusammengetragen. Dazu mussten insbesondere die älteren Jahrgänge nicht selten von Hand aus Hardcopies übertragen werden.

Die in der Datenbank erfassten Informationen umfassen neben der Konferenz und dem Jahr den Session Titel, den Paper Titel, die Autoren, deren Organisation sowie die von den Autoren angegebenen Keywords und das Abstract (letztere beide, sofern erhältlich). Als deutschen Ursprungs wurde ein Paper dann angesehen, wenn der Erstautor eine deutsche Institution/private Adresse angegeben hat.

Zur Auswertung wurden qualitative und quantitative Methoden der Datenanalyse kombiniert. ${ }^{14}$ Die nachfolgend näher erläuterte Klassifizierung der Daten stützt sich auf das Grounded Theory Verfahren, ${ }^{15}$ das weitere statistische Auswertungen ermöglicht.

Entsprechend des Grounded Theory Verfahrens wurden zunächst bekannte Themenfelder (u. a. die Bezeichnungen von Sessions bzw. Themenfelder entsprechend der Konferenz-Call-for-Papers) den Perspektiven (und ihren Schnittstellen) zugeordnet, indem die beiden folgenden Fragen beantwortet wurden: „Für welche Akteursgruppe ist das Thema bzw. die hier behandelte Entscheidungssituation besonders relevant?“ bzw. alternativ „Wessen Entscheidung beeinflusst dieses Thematik insbesondere?“. Für die nachgelagerte (Zeilen-)Kategorisierung wurde berücksichtigt, ob ein Thema sich eher auf das BezugsOBJEKT (und wenn ja, auf welches) oder die AKTIVITÄT (und wenn ja, welche) bezieht.

Für die diesbezügliche Kodierung der Themenfelder wurden Memos der Perspektiven, RE Typen und RE Aktivitäten erstellt, auf Basis deren dann die während der Kodierung der einzelnen Forschungsbeiträge weiter hinzukommenden Themenfelder

\footnotetext{
${ }^{14}$ Vgl. Miles, M. B. und Huberman, M., Qualitative 1994 sowie Mayring, P., Inhaltsanalyse, 2010.

${ }^{15}$ Vgl. Strauss, A. und Corbin, J., Qualitative 2008.
} 


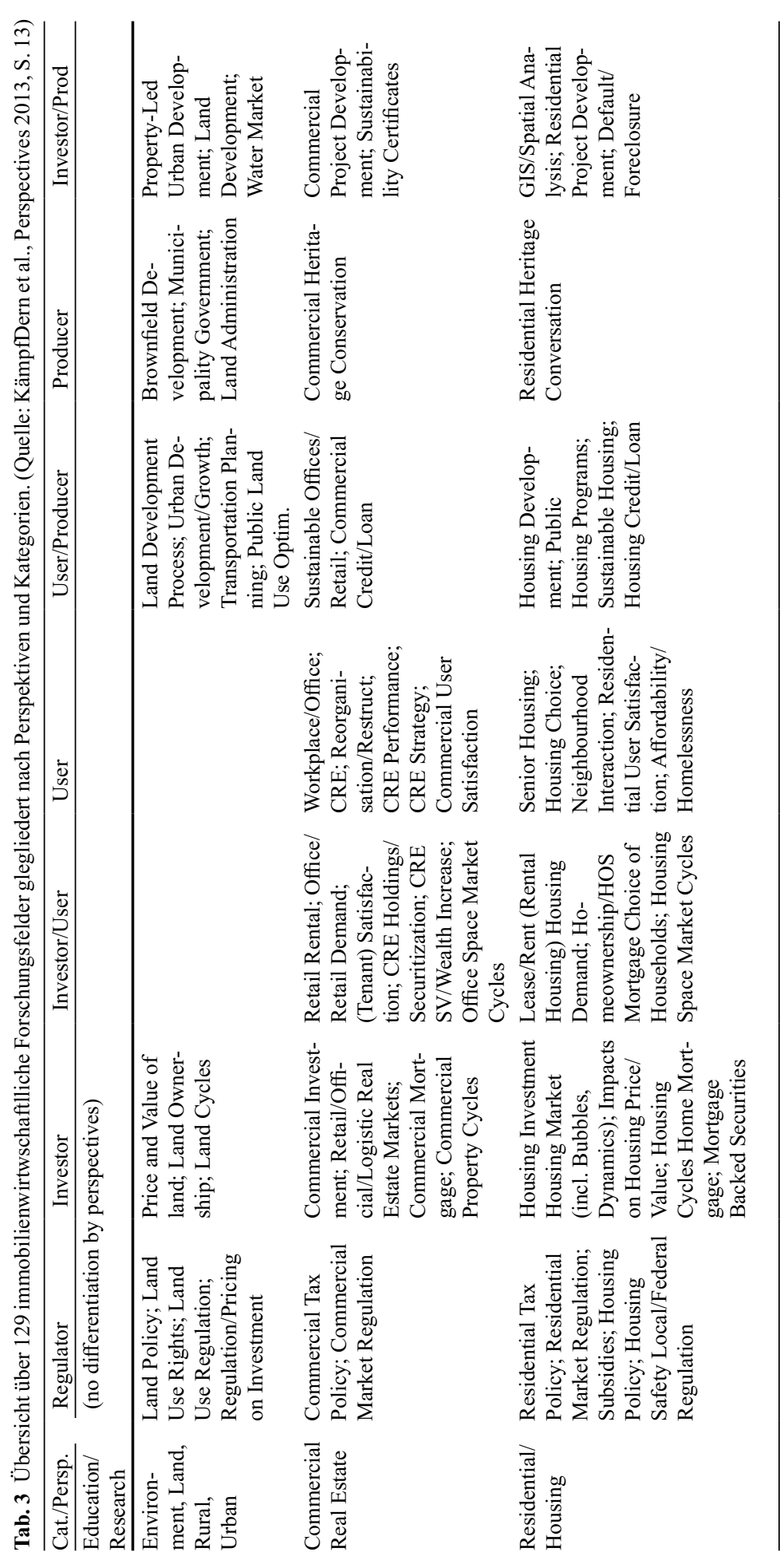




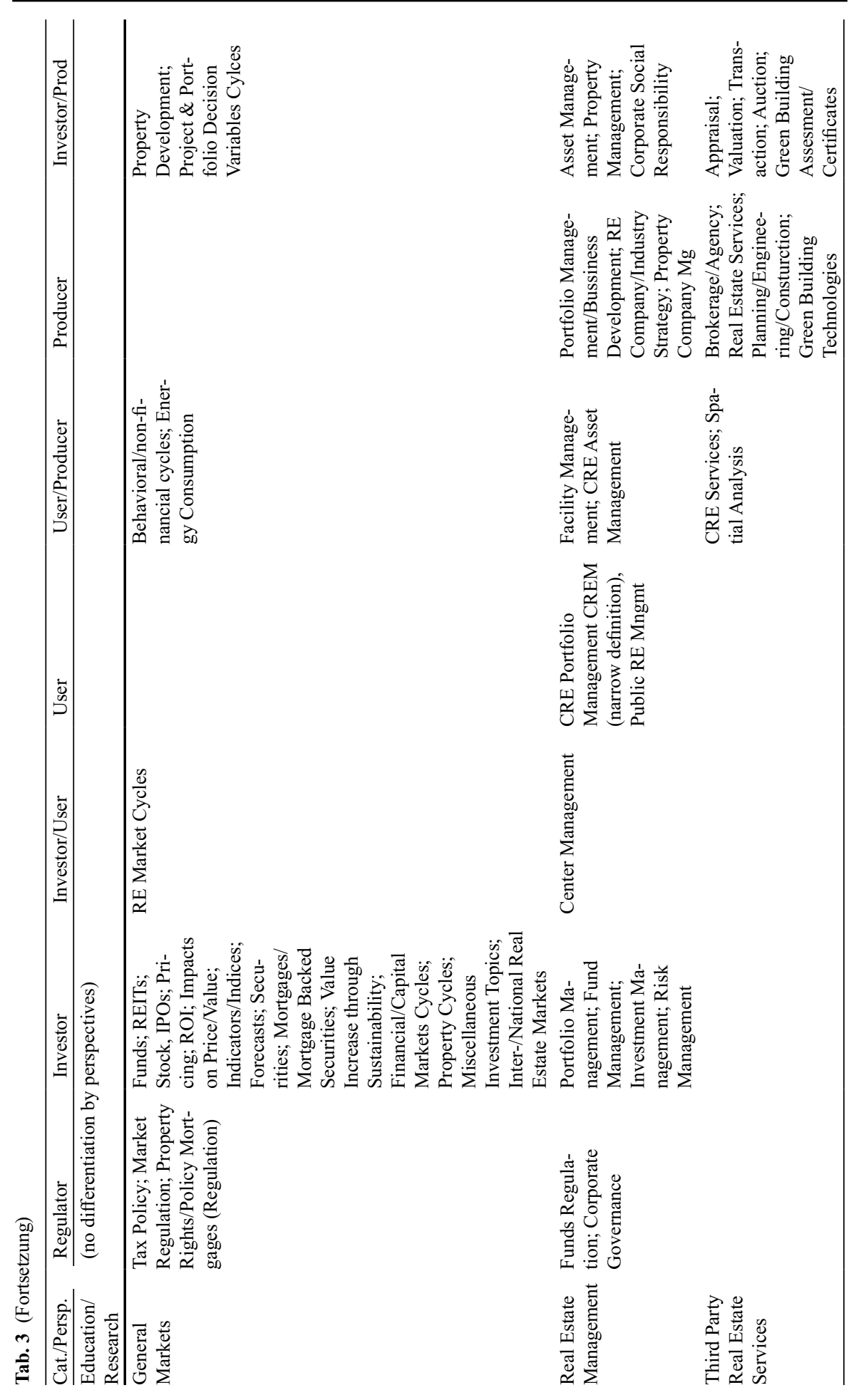


ebenfalls zugeordnet wurden. Durch dieses deduktiv und induktiv geleitete qualitative Vorgehen entstand somit eine Matrix mit vorerst 129 Themenfeldern (siehe Tab. 3).

Auf dieser Basis wurden entsprechend iterativ alle 14.124 Beiträge anhand ihrer Titel und - wo vorhanden - unter Berücksichtigung von Schlagworten und Abstracts nach Perspektive, Typ/Aktivität und Themenfeld kodiert.

3.2 Umfang des deutschen Anteils an der internationalen immobilienwirtschaftliche Forschung

Selektiert man die Forschungsbeiträge nach den von deutschen Erst-Autoren angefertigten Beiträgen, so zeigt sich das in Tab. 4 dargestellte Ergebnis:

Insgesamt wurden 533 (3,8\%) der 14.124 Beiträge von deutschen Autoren angefertigt. Der Großteil der Arbeiten wurde erwartungsgemäß auf den Konferenzen der ERES präsentiert. Hier waren deutsche Autoren an 372 (69,8\% aller deutschen Beiträge und 10,5\% der ERES Beiträge) beteiligt. Von zahlenmäßig größerer Bedeutung waren ferner die ARES Konferenzen mit 98 Beiträgen (18,4\% aller deutschen Beiträge und 2,9\% der ARES Beiträge) und die PRRES mit 26 Beiträgen (4,9\% aller deutschen Beiträge und 2,1\% der PRRES Beiträge).

Betrachtet man den Umfang des deutschen Anteils an internationalen Immobilienkonferenzen in Zeitscheiben von Dekaden, so hat sich die absolute Zahl der Beiträge von 90 im Zeitraum 1993 bis 2002 auf 443 in der zweiten Dekade von 2003 bis 2012 fast verfünffacht. Nach den USA, Australien, China und Großbritannien und vor Singapur und den Niederlanden hat die deutsche immobilienwirtschaftliche Forschung bislang weltweit den fünftgrößten Anteil aller Nationen. ${ }^{16}$ Wie die Abb. 2 zeigt, ist der relative Anteil an deutschen Forschungsbeiträgen auf internationalen Konferenzen steigend und liegt aktuell bei leicht über $5 \%$.

Die Ausreißer in der Datenreihe nach oben in den Jahren 1997 und 2006 sind dadurch begründet, dass in beiden Jahren ERES Konferenzen in Deutschland (1997 Berlin, 2006 Weimar) stattfanden. Unabhängig vom Austragungsort steigt der lineare Trend deutscher Beiträge auf ERES Veranstaltungen, die die einzigen hier untersuchten europäischen Konferenzen ausrichtet, in den letzten Jahren von unter 5\% auf knapp unter $15 \%$ an.

\subsection{Inhaltliche Schwerpunkte der immobilienwirtschaftlichen Forschung in Deutschland}

Im Folgenden stehen die Inhalte der deutschen immobilienwirtschaftlichen Forschung im Mittelpunkt des Interesses. Ausgehend von dem oben erläuterten Perspektiven-Modell der Immobilienwirtschaft verteilen sich die Inhalte der Paper wie folgt (Abb. 3):

In die Kategorie Perspektiven-übergreifender Forschung sind nachfolgend Arbeiten eingeordnet, die in ihren Problemstellungen zwei oder alle drei Perspektiven auf

\footnotetext{
${ }^{16}$ Dabei ist berücksichtigt, dass bislang bei ca. 1/3 der ERES-Beiträge die Autoren noch nicht Institutionen und damit Nationen zugeordnet werden konnten, so dass diesbezüglich eine Hochrechnung anhand der $2 / 3$ der zuordenbaren Beiträge erfolgte.
} 


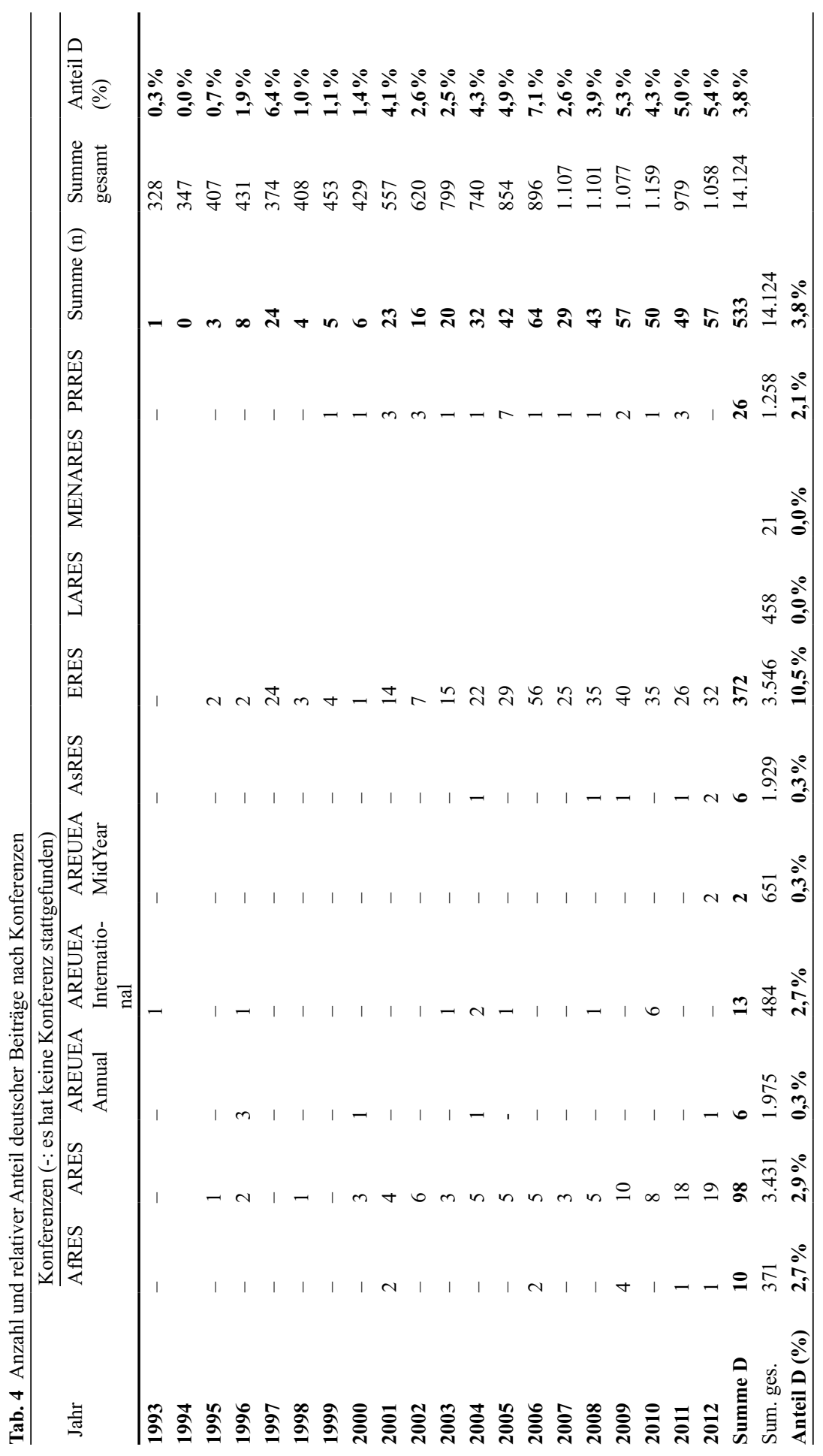




\section{Deutscher Anteil gesamt $(n=14.124)$}

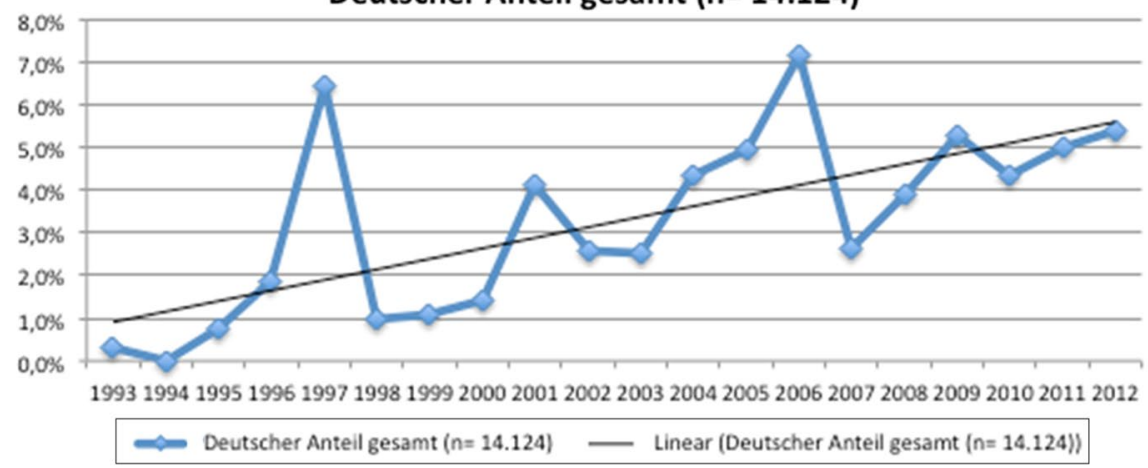

\section{Deutscher Anteil ERES ( $n=3.546)$}

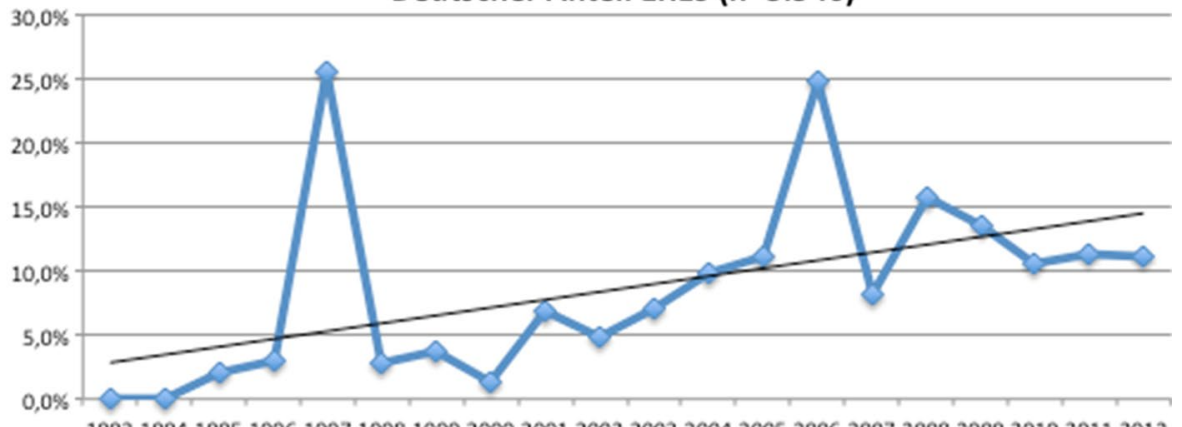

19931994199519961997199819992000200120022003200420052006200720082009201020112012 $\Longrightarrow$ Deutscher Anteil ERES $(n=3.546) \quad$ Linear (Deutscher Anteil ERES $(n=3.546))$

Abb. 2 Prozentualer Anteil deutscher Autoren im Zeitablauf

Abb. 3 Verteilung der Beiträge nach immobilienwirtschaftlichen Perspektiven $(N=533)$

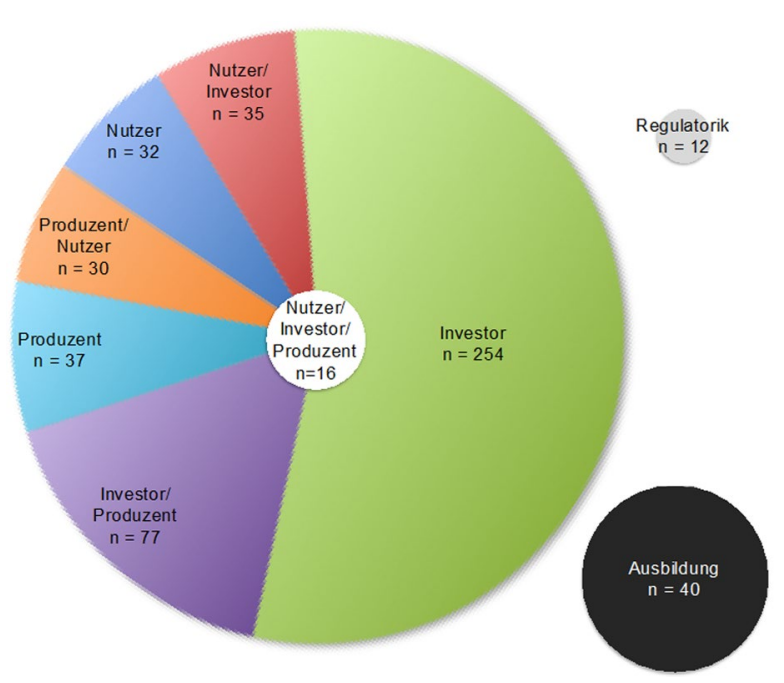


Tab. 5 Verteilung der Beiträge Perspektiven immobilienwirtschaftlicher Forschung

\begin{tabular}{|c|c|c|c|c|c|c|}
\hline \multirow[t]{2}{*}{ Perspektive } & \multicolumn{2}{|c|}{ Singulärer Fokus } & \multicolumn{2}{|c|}{ Perspektiven übergreifend } & \multicolumn{2}{|c|}{ Summe } \\
\hline & $\mathrm{n}$ & $\%(\mathrm{~N})$ & $\mathrm{n}$ & $\%(\mathrm{~N})$ & $\mathrm{n}$ & $\%(\mathrm{~N})$ \\
\hline Immobilien-nutzung & 32 & $6,0 \%$ & 81 & $15,2 \%$ & 113 & $21 \%$ \\
\hline Immobilien-investment & 254 & $47,7 \%$ & 128 & $24,0 \%$ & 382 & $72 \%$ \\
\hline $\begin{array}{l}\text { Produktion von Immobilien und } \\
\text { im. Dienstleistungen }\end{array}$ & 37 & $6,9 \%$ & 123 & $23,1 \%$ & 160 & $30 \%$ \\
\hline Regulatorik & 12 & $2,3 \%$ & & & 12 & $2 \%$ \\
\hline Ausbildung & 40 & $7,5 \%$ & & & 40 & $8 \%$ \\
\hline Summe & 375 & $70,4 \%$ & 332 & & & \\
\hline$N$ & 533 & & & & & \\
\hline
\end{tabular}

Tab. 6 Vergleich der Inhalte deutscher und globaler immobilienwirtschaftlicher Forschung

\begin{tabular}{|c|c|c|c|c|}
\hline & \multicolumn{2}{|c|}{ Deutschland } & \multicolumn{2}{|l|}{ Global } \\
\hline & Singulär & $\begin{array}{l}\text { Perspektiven } \\
\text { Übergreifend }\end{array}$ & Singulär & $\begin{array}{l}\text { Perspektiven } \\
\text { Übergreifend }\end{array}$ \\
\hline Perspektive & $\%(\mathrm{~N} \mathrm{D})$ & $\%(\mathrm{~N} \mathrm{D})$ & $\%$ (N global) & $\%(\mathrm{~N}$ global $)$ \\
\hline Immobiliennutzung & $6,0 \%$ & $15,2 \%$ & $6,3 \%$ & $15,3 \%$ \\
\hline Immobilieninvestment & $47,7 \%$ & $24,0 \%$ & $47,3 \%$ & $23,1 \%$ \\
\hline $\begin{array}{l}\text { Produktion von Immobilien und } \\
\text { im. Dienstleistungen }\end{array}$ & $6,9 \%$ & $23,1 \%$ & $6,5 \%$ & $23,1 \%$ \\
\hline Regulatorik & $2,3 \%$ & & $6,2 \%$ & \\
\hline Ausbildung & $7,5 \%$ & & $3,6 \%$ & \\
\hline$N$ & 533 & & 14.124 & \\
\hline
\end{tabular}

die Immobilie vereint adressieren. Dies ist zum Beispiel der Fall, wenn in einem Paper die Auswirkungen von Maßnahmen im Property Management (Produzenten Perspektive) auf die Rendite der Immobilie (Investoren Perspektive) analysiert wird.

Das in der Tab. 5 dargestellte Ergebnis zeigt, dass sich mit $72 \%$ der weit überwiegende Teil der Arbeiten direkt oder Perspektiven-übergreifend mit der Investorenperspektive auf die Immobilie auseinandersetzt.

Arbeiten, die Themenstellungen aus der Produzentenperspektive zum Gegenstand haben, sind mit einem Anteil von 30\% deutlich seltener. Am seltensten sind mit $21 \%$ der 533 Beiträge Forschungsarbeiten, die Problemstellungen aus der Immobiliennutzerperspektive thematisieren. Darüber hinaus befassen sich $8 \%$ aller Arbeiten mit Themen rund um die Immobilienausbildung und 2\% der Arbeiten mit Fragen der immobilienbezogenen Regulatorik.

\subsection{Vergleich der deutschen und internationalen Forschungsinhalte}

Der Vergleich der deutschen mit der globalen Intensität der Bearbeitung von Forschungsthemen in Tab. 6 zeigt, dass die Verteilung der perspektivischen Ausrichtung der deutschen Forschung mit der globalen Verteilung bis auf zehntel Prozentpunkte identisch ist.

Das betrifft sowohl die singulär aus einzelnen Perspektiven bearbeiteten Forschungsprobleme wie auch die perspektivenübergreifenden Anteile. Rechnerisch beträgt die Korrelation $0,99\left(r^{2}=0,98\right)$. Signifikante Unterschiede zeigen sich ledig- 
lich in den Bereichen Regulatorik und Ausbildung. Regulatorische Fragestellungen werden nur in 2,3\% der deutschen Arbeiten angesprochen, wohingegen sie global einen Anteil von 6,2\% aller Arbeiten einnehmen. Im Bereich der Ausbildung sind deutsche Forscher mit 7,5\% aller Arbeiten doppelt so stark engagiert als es dem globalen Anteil von 3,6\% entsprechen würde. Dieses nahezu identische Ergebnis der deutschen mit den globalen Arbeitsschwerpunkten ist sehr überraschend, da die Auswertungen unterschiedlicher Konferenzen sehr große Abweichungen gezeigt haben. ${ }^{17}$ So sind insbesondere ARES und PRRES Konferenzen sehr viel stärker investmentspezifisch, wohingegen insbesondere die ERES Konferenz sich durch eine stärkere Ausgewogenheit der Themen kennzeichnet. Da die deutschen Wissenschaftler für ihre Präsentationen die ERES Konferenz bevorzugen, wäre zu erwarten gewesen, dass sich die größere Ausgewogenheit der ERES gegenüber den anderen großen Konferenzen auch in den deutschen Forschungsschwerpunkten niederschlägt. Stattdessen muss auf der Basis der Ergebnisse schlussgefolgert werden, dass es nicht die deutschen Forscher sind, die bei der ERES-Konferenz themen-diversifizierend wirken.

\subsection{Themenstellungen immobilienwirtschaftlicher Forschung in Deutschland}

Jeder Versuch der weiteren Aufgliederung von Forschungsinhalten, der über die oben angestellte perspektivische Betrachtung hinausgeht, muss das Problem lösen, dass immobilienwirtschaftliche Fragestellungen im wissenschaftlichen Prozess bislang nur in sehr wenigen Teilbereichen theoretisch trennscharf abgegrenzt wurden oder eindeutigen Literatursträngen zurechenbar sind. Vielmehr orientiert sich die Mehrzahl der Forschungsarbeiten an empirischen Problemsichten, die - wie man der oben aufgeführten Themenvielfalt entnehmen kann - wesentlich breiter gestreut sind und oft Bezüge zu mehreren Theoriegebieten aufweisen und diese verknüpfen. Deshalb wurden alle 533 immobilienwirtschaftlichen Forschungsarbeiten durch eine detaillierte Analyse der Titel, Keywords und Abstracts nicht nach immobilienwirtschaftlichen Theorien über den Perspektivenansatz hinaus, sondern nach entsprechend ermitteltem Matrix-Bezugsrahmen kodiert und unter Zuhilfenahme der Kategorienmemos und der fortgeschriebenen Themenliste jeweils einem Thema händisch zugeordnet. Wie bereits dargestellt bestehen dabei Überschneidungsbereiche, so dass ein Beitrag gegebenenfalls zwei oder mehreren Themen bzw. Matrix-Zellen zugeordnet werden könnte. Darauf wurde jedoch zugunsten einer eindeutigen Zuordnung bewusst verzichtet. Dies erscheint nicht nur angesichts der relativ hohen Anzahl an Dokumenten und einer daraus folgenden Robustheit der Daten als auch insofern vertretbar, dass die Zuordnungen memobasiert erfolgten, für jeden Einzelfall dokumentiert und damit jederzeit nachvollziehbar sowie notfalls adaptierbar sind.

Da die Themenbereiche jedoch sehr unterschiedliche Detaillierungsgrade aufweisen, ist bei der Interpretation der Ergebnisse insofern bezüglich der rein quantitativen Aussagen Vorsicht geboten: Während sich die Forschung im Bereich der Investmentperspektiven bereits in zahlreiche einzelne Themenbereiche zergliedert, sind in der Produzenten- und der Nutzerperspektive erst vergleichsweise weniger und breitere

${ }^{17}$ Vgl. Kämpf-Dern, A. et al., Perspectives, 2013. 
Themenbereiche erkennbar, die mehrfach bearbeitet wurden. In der nachfolgenden Tab. 7 sind die TOP-50 Forschungsthemen nach der Anzahl an Beiträgen in absteigender Reihenfolge aufgeführt.

Auffällig ist, dass deutsche Forscher sich (in 29 Beiträgen, 5,4\%) am stärksten mit der Erforschung ihres eigenen Arbeitsalltags auseinandersetzen. An den nächsten Stellen folgen mit Beiträgen über REITs (2.), Immobilienbewertung (3.) sowie diverse Immobilienmarkt- und Immobilieninvestmentthemen (4.-7.) deutlich spezifischere Betrachtungen. Bemerkenswert ist, dass klassische Immobilienthemen wie Wohnungswirtschaft, Corporate Real Estate Management, Wertermittlung sowie Transaktionen vergleichsweise weniger starke Berücksichtigung gefunden haben. Im Großen und Ganzen sind diese Ergebnisse kompatibel zu den Ergebnissen der von Schulte et al. (2004) durchgeführten Befragung deutscher Wissenschaftler zu den wichtigsten Forschungsgebieten, wenngleich eine direkte Vergleichbarkeit aufgrund terminlicher, methodischer und vor allem inhaltlich-struktureller Unterschiede der empirischen Untersuchungen nicht möglich ist. ${ }^{18}$

Die Tatsache, dass die geringe Anzahl von drei Forschungsarbeiten zu einem Forschungsthema ausreicht, um damit einen TOP-50 Platz einzunehmen, verdeutlicht einen geringen Konzentrationsgrad der Forschung. Unter die TOP-25 fällt ein Forschungsgebiet bereits mit 7 Konferenzbeiträgen und unter die TOP-10 mit 16 Beiträgen, wohlgemerkt jeweils in 20 Jahren auf bis zu 10 Konferenzen pro Jahr.

Um die Selektion deutscher Forschungsthemen im Allgemeinen und den vergleichsweise geringen Konzentrationsgrad der deutschen immobilienwirtschaftlichen Konferenzbeiträge im Besonderen weiter beurteilen zu können, ist ein Abgleich mit dem globalen Bestand an Forschungsbeiträgen zweckdienlich. Die Tab. 8 zeigt die Häufigkeit von Konferenzbeiträgen zu zwei Drittel der am häufigsten frequentierten Forschungsthemen.

Global entfallen zwei Drittel (9283) aller Konferenzbeiträge auf 34 unterschiedliche Themenbereiche, während in Deutschland 26 Themengebiete zwei Drittel aller Konferenzbeiträge auf sich vereinen. Das bedeutet, dass die Forschungsthemen in Deutschland konzentrierter sind als im weltweiten Durchschnitt. In der folgenden Tab. 9 sind die Top-25 nebeneinandergestellt.

Die Korrelation zwischen der Verteilung deutscher und globaler Konferenzbeiträge auf die jeweiligen Forschungsthemen beträgt 0,70, das Bestimmtheitsmaß $\mathrm{r}^{2}$ beträgt 0,48 . Der lineare Zusammenhang der beiden Datenreihen kann somit als mittelstark eingestuft werden. Die Statistik bestätigt damit, dass die deutsche Forschung in einem gewissen Maß abhängig von dem globalen Geschehen ist. Da die deutsche Forschung in den globalen Umfang, wenn auch nur zu einem recht geringen Maße, unmittelbar einfließt und alle Forscher auf den Konferenzen im regen Austausch sind, war dieses Ergebnis zu erwarten, wenn auch nicht unbedingt in dieser Deutlichkeit.

Die qualitativ vergleichende Analyse der Top-25 Themen bestätigt das oben erzielte Ergebnis, dass die inhaltliche Struktur deutscher Konferenzbeiträge recht weitgehend dem globalen Bild immobilienwirtschaftlicher Forschung entspricht. Unterschiede ergeben sich bei einer relativ gesehen stärkeren Beachtung der Themenfelder Education, CREM und Property Development in der deutschen Forschung. Im globalen

${ }^{18}$ Vgl. Schulte, K.-W. et al., Forschungsschwerpunkte 2004, insb. S. 12 ff. 
Tab. 7 Top-50 Inhalte deutscher Konferenzbeiträge nach primären Themenstellungen

\begin{tabular}{|c|c|c|c|c|}
\hline $\mathrm{Rg}$. & Deutsche Übersetzung & Englische Bezeichnung (Original) & $\mathrm{n}$ & $\%$ \\
\hline 1 & Forschung und Lehre & Education & 29 & $5,4 \%$ \\
\hline 2 & REITs & REITs & 28 & $5,3 \%$ \\
\hline 3 & Immobilienbewertung & Valuation (Models/Methods) & 24 & $4,5 \%$ \\
\hline 4 & Gewerbeimmobilienmärkte & $\begin{array}{l}\text { Retail/Office/Logistic Real Estate } \\
\text { Markets }\end{array}$ & 22 & $4,1 \%$ \\
\hline 5 & Div. Immobilieninvestmentthemen & Miscellaneous Investment Topics & 22 & $4,1 \%$ \\
\hline 6 & Wohnimmobilienmärkte & $\begin{array}{l}\text { Housing Market (incl. Bubbles, } \\
\text { Dynamics) }\end{array}$ & 21 & $3,9 \%$ \\
\hline 7 & (Inter)Nationale Immobilienmärkte & Inter-/National Real Estate Markets & 21 & $3,9 \%$ \\
\hline 8 & Immobilienfonds & Funds & 20 & $3,8 \%$ \\
\hline 9 & Portfolio Management & Portfolio Management & 17 & $3,2 \%$ \\
\hline 10 & Verbriefung von Immobilieneigentum & Securities & 16 & $3,0 \%$ \\
\hline 10 & Risikomanagement & Risk Management & 16 & $3,0 \%$ \\
\hline 12 & Öffentlich Private Partnerschaften & Public Private Partnership & 12 & $2,3 \%$ \\
\hline 13 & Immobilienzyklen & Real Estate Pricing/Market Cycles & 11 & $2,1 \%$ \\
\hline 14 & Marktforschung & Research & 10 & $1,9 \%$ \\
\hline 14 & Immobilienentwicklung allgemein & Property Development & 10 & $1,9 \%$ \\
\hline 16 & Wohnungsinvestments & Housing Investment & 9 & $1,7 \%$ \\
\hline 16 & Immobilienaktien, IPOs & Stock, IPOs & 9 & $1,7 \%$ \\
\hline 16 & $\begin{array}{l}\text { Corporate Real Estate Management } \\
\text { i.e.S. }\end{array}$ & $\begin{array}{l}\text { CREM (narrow definition), Non-Pro- } \\
\text { perty Companies RE Mgmt. }\end{array}$ & 9 & $1,7 \%$ \\
\hline 16 & Nachhaltige Wohnungswirtschaft & Sustainable Housing & 9 & $1,7 \%$ \\
\hline 20 & Investment Management & Investment Management & 8 & $1,5 \%$ \\
\hline 20 & Unternehmensstrategie & RE Company/Industry Strategy & 8 & $1,5 \%$ \\
\hline 20 & Immobilienservices & Real Estate Services & 8 & $1,5 \%$ \\
\hline 23 & Immobilienwertermittlung & Appraisal & 7 & $1,3 \%$ \\
\hline 23 & Selbstgenutztes Wohneigentum & Homeownership/HOS & 7 & $1,3 \%$ \\
\hline 23 & Immobilienrenditen & ROI & 7 & $1,3 \%$ \\
\hline 23 & $\begin{array}{l}\text { Nachfrage nach } \\
\text { Gewerbeimmobilienflächen }\end{array}$ & Office/Retail Demand & 7 & $1,3 \%$ \\
\hline 27 & Stadtentwicklung & Urban Development/Growth & 6 & $1,1 \%$ \\
\hline 27 & Indikatoren und Indizes & Indicators/Indices & 6 & $1,1 \%$ \\
\hline 27 & Wohnungsvermietung & Lease/Rent (Rental Housing) & 6 & $1,1 \%$ \\
\hline 27 & Transaktionen & Transaction & 6 & $1,1 \%$ \\
\hline 27 & Landentwicklung & Land Development & 6 & $1,1 \%$ \\
\hline 32 & Geschäftsfeldentwicklung & $\begin{array}{l}\text { Portfolio Management/Business } \\
\text { Development }\end{array}$ & 5 & $0,9 \%$ \\
\hline 32 & Corporate Governance & Corporate Governance & 5 & $0,9 \%$ \\
\hline 32 & Asset Management & Asset Management & 5 & $0,9 \%$ \\
\hline 32 & Arbeitsplatzgestaltung & Workplace/Office & 5 & $0,9 \%$ \\
\hline 36 & $\begin{array}{l}\text { Management von } \\
\text { Immobilienunternehmen }\end{array}$ & Property Company Mngmt & 4 & $0,8 \%$ \\
\hline 36 & Gewerbliches Immobilienkreditgeschäft & $\begin{array}{l}\text { Mortgages/Mortgage Backed } \\
\text { Securities }\end{array}$ & 4 & $0,8 \%$ \\
\hline 36 & Werteffekte von Nachhaltigkeit & Value Increase through Sustainability & 4 & $0,8 \%$ \\
\hline 36 & Green Building Technologien & Green Building Technologies & 4 & $0,8 \%$ \\
\hline 36 & $\begin{array}{l}\text { Entscheidungsdeterminanten bei Pro- } \\
\text { jektentwicklungen, Flächennachfrage }\end{array}$ & PPDV Cycles User/Producer & 4 & $0,8 \%$ \\
\hline
\end{tabular}


Tab. 7 (Fortsetzung)

\begin{tabular}{lllll}
\hline Rg. & Deutsche Übersetzung & Englische Bezeichnung (Original) & $\mathrm{n}$ & $\%$ \\
\hline 36 & Wohnimmobilienentwicklung & Residential Project Development & 4 & $0,8 \%$ \\
36 & Bezahlbarer Wohnraum & Affordability/Homelessness & 4 & $0,8 \%$ \\
36 & Öffentliche Wohnraumförderung & Public Housing Programs & 4 & $0,8 \%$ \\
44 & Preis und Wertdeterminanten & Impacts on Price/Value & 3 & $0,6 \%$ \\
44 & Projektentwicklung von & Commercial Project Development & 3 & $0,6 \%$ \\
& Gewerbeimmobilien & & \\
44 & Wohnungskreditgeschäft & Home Mortgage & 3 & $0,6 \%$ \\
44 & Entscheidungsvariablen am Investment- & PPDV Cycles Investor/Producer & 3 & $0,6 \%$ \\
& markt für Entwickler & & 3 & $0,6 \%$ \\
44 & Corporate Real Estate Performance & CRE Performance & 3 & $0,6 \%$ \\
44 & Wohnungspolitik & Housing Policy & 3 & $0,6 \%$ \\
44 & Corporate Real Estate & CRE & 3 & $0,6 \%$ \\
44 & Center Management & Center Management & 3 & $0,6 \%$ \\
44 & Nachhaltigkeit, Zertifikate & Sustainability Certificates & 3 & $0,6 \%$ \\
44 & Nutzerzufriedenheit, Gewerbeflächen & Commercial User Satisfaction & & \\
\hline
\end{tabular}

Vergleich relativ geringer fallen hingegen die Gewichte der Analyse von Marktzyklen (Cycles), verschiedener wohnungswirtschaftlicher Themen wie u. a. Home Mortgage oder Impact on Housing Price/Value sowie die Bereiche Stadtentwicklung (Urban Development) und Maklerwesen (Brokerage/Agency) aus.

Aus der vergleichenden Analyse werden ferner die Domänen der deutschen Immobilienwirtschaftlichen Forschung deutlich. Die Tab. 10 zeigt alle Themengebiete in absteigender Reihenfolge, in denen deutsche Forscher mehr als 5\% der globalen Konferenzbeiträge präsentiert haben.

Die deutschen Konferenzbeiträge haben den größten Anteil an Themenbereichen zu nachfrageseitigen Immobilienmarktzyklen am Flächenmarkt (RE Rental Demand Cycles und PPDV Cycles User/Producer), Public Private Partnership, Asset Management sowie Corporate Real Estate Management (CRE Performance, CRE SV/Wealth Increase). Bei all diesen Bereichen handelt es sich um global weniger stark behandelte Themen. Selektiert man die Liste nach Themen, die weltweit mindestens 1,0\% aller Forschungsthemen auf sich vereinen, so zeigen sich Schwerpunkte deutscher Forschung in den Themenbereichen Portfoliomanagement, Education, Securities, Research und Stock, IPOs.

Um den Einfluss der deutschen Konferenzbeiträge auf die globale Forschung zu veranschaulichen, können die Gewichte der jeweiligen Forschungsthemen (als Anteil in \% von N (Global)) mit dem Anteil der deutschen Forschung an dem Thema multipliziert werden. Diesem Vorgehen liegt die Prämisse zugrunde, dass mit zunehmender Anzahl an globalen Konferenzbeiträgen auch die Bedeutung des Themas für die Forschungslandschaft steigt. Durch Multiplikation mit 10.000, um unnötige Nullstellen bereinigt, ergibt sich ein Wert, der mit aller Vorsicht als eine Art Impact Factor des deutschen Anteils an bestimmten Forschungsthemen interpretiert werden kann. Die Tab. 11 zeigt in absteigender Reihenfolge die Top- 25 Themen.

Die Auswertung zeigt, dass deutsche Forscher über die Bereiche Education, REITs und Bewertung am stärksten auf die internationalen Konferenzen Einfluss genommen haben. In den Top-10 folgen Immobilienmarkt- und -Investmentthemen, Fondsthe- 
Tab. 8 Inhalte globaler Konferenzbeiträge nach häufigsten Themenstellungen

\begin{tabular}{lllll}
\hline Rg. & Themengebiet & $\mathrm{n}$ & $\%(\mathrm{~N})$ & $\% \mathrm{kum}$. \\
\hline 1 & Housing Market (incl. Bubbles, Dynamics) & 999 & $7,1 \%$ & $7,1 \%$ \\
2 & REITs & 723 & $5,1 \%$ & $12,2 \%$ \\
3 & Valuation (Models/Methods) & 671 & $4,8 \%$ & $16,9 \%$ \\
4 & Retail/Office/Logistic Real Estate Markets & 519 & $3,7 \%$ & $20,6 \%$ \\
5 & Inter-/National Real Estate Markets & 430 & $3,0 \%$ & $23,7 \%$ \\
6 & Real Estate Pricing/Market Cycles & 386 & $2,7 \%$ & $26,4 \%$ \\
7 & Home Mortgage & 383 & $2,7 \%$ & $29,1 \%$ \\
8 & Impacts on Housing Price/Value & 341 & $2,4 \%$ & $31,5 \%$ \\
9 & Education & 332 & $2,4 \%$ & $33,9 \%$ \\
10 & Miscellaneous Investment Topics & 323 & $2,3 \%$ & $36,2 \%$ \\
11 & Urban Development/Growth & 306 & $2,2 \%$ & $38,3 \%$ \\
12 & Brokerage/Agency & 245 & $1,7 \%$ & $40,1 \%$ \\
13 & Appraisal & 241 & $1,7 \%$ & $41,8 \%$ \\
14 & Mortgages/Mortgage Backed Securities & 235 & $1,7 \%$ & $43,4 \%$ \\
15 & Homeownership/HOS & 234 & $1,7 \%$ & $45,1 \%$ \\
16 & Housing Investment & 222 & $1,6 \%$ & $46,7 \%$ \\
17 & Securities & 193 & $1,4 \%$ & $48,0 \%$ \\
18 & Indicators/Indices & 190 & $1,3 \%$ & $49,4 \%$ \\
19 & Portfolio Management & 177 & $1,3 \%$ & $50,6 \%$ \\
20 & Housing Choice & 176 & $1,2 \%$ & $51,9 \%$ \\
21 & Default/Foreclosure & 175 & $1,2 \%$ & $53,1 \%$ \\
22 & Housing Policy & 171 & $1,2 \%$ & $54,3 \%$ \\
22 & Affordability/Homelessness & 171 & $1,2 \%$ & $55,5 \%$ \\
24 & Impacts on Price/Value & 164 & $1,2 \%$ & $56,7 \%$ \\
25 & Residential Project Development & 161 & $1,1 \%$ & $57,8 \%$ \\
26 & Price and Value of Land & 155 & $1,1 \%$ & $58,9 \%$ \\
27 & Lease/Rent (Rental Housing) & 149 & $1,1 \%$ & $60,0 \%$ \\
28 & Research & 139 & $1,0 \%$ & $61,0 \%$ \\
29 & Stock, IPOs & 138 & $1,0 \%$ & $61,9 \%$ \\
29 & Housing Demand & 138 & $1,0 \%$ & $62,9 \%$ \\
31 & Risk Management & 134 & $0,9 \%$ & $63,9 \%$ \\
32 & ROI & 132 & $0,9 \%$ & $64,8 \%$ \\
33 & Funds & 130 & $0,9 \%$ & $65,7 \%$ \\
34 & Tax Policy & 127 & $0,9 \%$ & $66,6 \%$ \\
\hline & & & & \\
& & & \\
& & &
\end{tabular}

men, Portfoliomanagement und Securities. Es fällt ferner auf, dass die ersten sieben Plätze dieses Impact Rankings von Forschungsgebieten belegt wurde, die im globalen Ranking der meistbearbeiteten Themen einen Top-10 Platz einnehmen.

\subsection{Wo wurden in Deutschland immobilienwirtschaftliche Beiträge für internationale Konferenzen erstellt?}

Zur Analyse der Organisationen, an denen in Deutschland immobilienwirtschaftliche Forschungsbeiträge für internationale Konferenzen erstellt wurden, sind alle 533 deutschen Beiträge nach dem erstgenannten Autor seiner angegebenen oder zuletzt 
Tab. 9 Top-25 Themenstellungen Global und D im Vergleich

\begin{tabular}{|c|c|c|c|c|c|c|c|c|}
\hline$\overline{\mathrm{Rg}}$. & Themengebiet & $\mathrm{n}$ & $\%$ & Themengebiet & $\mathrm{n}$ & $\%$ & $\begin{array}{l}\text { Rg. } \\
\text { Global }\end{array}$ & $\begin{array}{l}\text { Anteil } \\
\text { Global }\end{array}$ \\
\hline 1 & $\begin{array}{l}\text { Housing Market } \\
\text { (incl. Bubbles, } \\
\text { Dynamics) }\end{array}$ & 999 & $7,1 \%$ & Education & 29 & $5,5 \%$ & 9 & $8,7 \%$ \\
\hline 2 & REITs & 732 & $5,2 \%$ & REITs & 28 & $5,3 \%$ & 2 & $3,8 \%$ \\
\hline 3 & $\begin{array}{l}\text { Valuation (Models/ } \\
\text { Methods) }\end{array}$ & 671 & $4,8 \%$ & Valuation (Models/Methods) & 24 & $4,6 \%$ & 3 & $3,6 \%$ \\
\hline 4 & $\begin{array}{l}\text { Retail/Office/Lo- } \\
\text { gistic Real Estate } \\
\text { Markets }\end{array}$ & 519 & $3,7 \%$ & $\begin{array}{l}\text { Retail/Office/Logistic Real } \\
\text { Estate Markets }\end{array}$ & 22 & $4,2 \%$ & 4 & $4,2 \%$ \\
\hline 5 & $\begin{array}{l}\text { Inter-/National Real } \\
\text { Estate Markets }\end{array}$ & 430 & $3,0 \%$ & $\begin{array}{l}\text { Miscellaneous Investment } \\
\text { Topics }\end{array}$ & 22 & $4,2 \%$ & 10 & $6,8 \%$ \\
\hline 6 & $\begin{array}{l}\text { Real Estate Pricing/ } \\
\text { Market Cycles }\end{array}$ & 386 & $2,7 \%$ & $\begin{array}{l}\text { Housing Market (incl. Bub- } \\
\text { bles, Dynamics) }\end{array}$ & 21 & $4,0 \%$ & 1 & $2,1 \%$ \\
\hline 7 & Home Mortgage & 383 & $2,7 \%$ & $\begin{array}{l}\text { Inter-/National Real Estate } \\
\text { Markets }\end{array}$ & 21 & $4,0 \%$ & 5 & $4,9 \%$ \\
\hline 8 & $\begin{array}{l}\text { Impacts on Housing } \\
\text { Price/Value }\end{array}$ & 341 & $2,4 \%$ & Funds & 20 & $3,8 \%$ & 33 & $15,4 \%$ \\
\hline 9 & Education & 332 & $2,4 \%$ & Portfolio Management & 17 & $3,2 \%$ & 19 & $9,6 \%$ \\
\hline 10 & $\begin{array}{l}\text { Miscellaneous } \\
\text { Investment Topics }\end{array}$ & 323 & $2,3 \%$ & Securities & 16 & $3,0 \%$ & 18 & $8,3 \%$ \\
\hline 11 & $\begin{array}{l}\text { Urban De- } \\
\text { velopment/ } \\
\text { Growth }\end{array}$ & 306 & $2,2 \%$ & Risk Management & 16 & $3,0 \%$ & 31 & $11,9 \%$ \\
\hline 12 & Brokerage/Agency & 245 & $1,7 \%$ & Public Private Partnership & 12 & $2,3 \%$ & 71 & $22,6 \%$ \\
\hline 13 & Appraisal & 241 & $1,7 \%$ & RE Rental Demand Cycles & 11 & $2,1 \%$ & 97 & $40,7 \%$ \\
\hline 14 & $\begin{array}{l}\text { Mortgages/ } \\
\text { Mortgage Backed } \\
\text { Securities }\end{array}$ & 235 & $1,7 \%$ & Research & 10 & $1,9 \%$ & 28 & $7,2 \%$ \\
\hline 15 & $\begin{array}{l}\text { Homeownership/ } \\
\text { HOS }\end{array}$ & 234 & $1,7 \%$ & Property Development & 10 & $1,9 \%$ & 37 & $8,1 \%$ \\
\hline 16 & Housing Investment & 222 & $1,6 \%$ & Housing Investment & 9 & $1,7 \%$ & 16 & $4,1 \%$ \\
\hline 17 & Indicators/Indices & 203 & $1,4 \%$ & Stock, IPOs & 9 & $1,7 \%$ & 30 & $6,5 \%$ \\
\hline 18 & Securities & 193 & $1,4 \%$ & $\begin{array}{l}\text { CREM (narrow definition), } \\
\text { Non-Property Companies RE } \\
\text { Mgmt. }\end{array}$ & 9 & $1,7 \%$ & 57 & $11,5 \%$ \\
\hline 19 & $\begin{array}{l}\text { Portfolio } \\
\text { Management }\end{array}$ & 177 & $1,3 \%$ & Sustainable Housing & 9 & $1,7 \%$ & 65 & $13,4 \%$ \\
\hline 20 & Housing Choice & 176 & $1,2 \%$ & Investment Management & 8 & $1,5 \%$ & 47 & $8,0 \%$ \\
\hline 21 & Default/Foreclosure & 175 & $1,2 \%$ & $\begin{array}{l}\text { RE Company/Industry } \\
\text { Strategy }\end{array}$ & 8 & $1,5 \%$ & 50 & $8,8 \%$ \\
\hline 22 & $\begin{array}{l}\text { Affordability/ } \\
\text { Homelessness }\end{array}$ & 171 & $1,2 \%$ & Real Estate Services & 8 & $1,5 \%$ & 52 & $9,0 \%$ \\
\hline 23 & Housing Policy & 171 & $1,2 \%$ & Appraisal & 7 & $1,3 \%$ & 13 & $2,9 \%$ \\
\hline 24 & $\begin{array}{l}\text { Impacts on Price/ } \\
\text { Value }\end{array}$ & 164 & $1,2 \%$ & Homeownership/HOS & 7 & $1,3 \%$ & 15 & $3,0 \%$ \\
\hline 25 & $\begin{array}{l}\text { Residential Project } \\
\text { Development }\end{array}$ & 161 & $1,1 \%$ & ROI & 7 & $1,3 \%$ & 32 & $5,3 \%$ \\
\hline
\end{tabular}


Tab. 10 Domänen deutscher immobilienwirtschaftlicher Forschung

\begin{tabular}{|c|c|c|c|c|c|c|}
\hline \multirow[t]{2}{*}{ Rg. } & \multirow[t]{2}{*}{ Themengebiet } & \multirow{2}{*}{$\begin{array}{l}\text { Anteil D an } \\
\text { Global } \\
\%\end{array}$} & \multicolumn{2}{|c|}{ Global } & \multicolumn{2}{|c|}{ Deutschland } \\
\hline & & & $\mathrm{n}$ & $\%$ & $\mathrm{n}$ & $\%$ \\
\hline 1 & RE Rental Demand Cycles & $40,7 \%$ & 27 & $0,2 \%$ & 11 & $2,1 \%$ \\
\hline 2 & PPDV Cycles User/Producer & $25,0 \%$ & 16 & $0,1 \%$ & 4 & $0,8 \%$ \\
\hline 3 & Public Private Partnership & $22,6 \%$ & 53 & $0,4 \%$ & 12 & $2,3 \%$ \\
\hline 4 & Asset Management & $16,7 \%$ & 30 & $0,2 \%$ & 5 & $1,0 \%$ \\
\hline 4 & CRE Performance & $16,7 \%$ & 18 & $0,1 \%$ & 3 & $0,6 \%$ \\
\hline 4 & CRE SV/Wealth Increase & $16,7 \%$ & 12 & $0,1 \%$ & 2 & $0,4 \%$ \\
\hline 4 & Housing Safety Regulations & $16,7 \%$ & 6 & $0,0 \%$ & 1 & $0,2 \%$ \\
\hline 8 & Corporate Governance & $16,1 \%$ & 31 & $0,2 \%$ & 5 & $1,0 \%$ \\
\hline 8 & $\begin{array}{l}\text { Portfolio Management/Business } \\
\text { Development }\end{array}$ & $16,1 \%$ & 31 & $0,2 \%$ & 5 & $1,0 \%$ \\
\hline 10 & Commercial User Satisfaction & $15,8 \%$ & 19 & $0,1 \%$ & 3 & $0,6 \%$ \\
\hline 10 & PPDV Cycles Investor/Producer & $15,8 \%$ & 19 & $0,1 \%$ & 3 & $0,6 \%$ \\
\hline 12 & Sustainability Certificates & $15,8 \%$ & 19 & $0,1 \%$ & 3 & $0,6 \%$ \\
\hline 13 & Funds & $15,4 \%$ & 130 & $0,9 \%$ & 20 & $3,8 \%$ \\
\hline 14 & Green Building Technologies & $14,8 \%$ & 27 & $0,2 \%$ & 4 & $0,8 \%$ \\
\hline 15 & Sustainable Housing & $13,4 \%$ & 67 & $0,5 \%$ & 9 & $1,7 \%$ \\
\hline 16 & $\begin{array}{l}\text { Value Increase through } \\
\text { Sustainability }\end{array}$ & $13,3 \%$ & 30 & $0,2 \%$ & 4 & $0,8 \%$ \\
\hline 17 & Risk Management & $11,9 \%$ & 134 & $0,9 \%$ & 16 & $3,0 \%$ \\
\hline 18 & $\begin{array}{l}\text { CREM (narrow definition), Non- } \\
\text { Property Companies REM }\end{array}$ & $11,5 \%$ & 78 & $0,6 \%$ & 9 & $1,7 \%$ \\
\hline 19 & Portfolio Management & $9,6 \%$ & 177 & $1,3 \%$ & 17 & $3,2 \%$ \\
\hline 20 & Sustainable Offices/Retail & $9,1 \%$ & 22 & $0,2 \%$ & 2 & $0,4 \%$ \\
\hline 21 & Real Estate Services & $9,0 \%$ & 89 & $0,6 \%$ & 8 & $1,5 \%$ \\
\hline 22 & RE Company/Industry Strategy & $8,8 \%$ & 91 & $0,6 \%$ & 8 & $1,5 \%$ \\
\hline 23 & Education & $8,7 \%$ & 332 & $2,4 \%$ & 29 & $5,5 \%$ \\
\hline 24 & Securities & $8,3 \%$ & 193 & $1,4 \%$ & 16 & $3,0 \%$ \\
\hline 25 & Property Development & $8,1 \%$ & 123 & $0,9 \%$ & 10 & $1,9 \%$ \\
\hline 26 & Center Management & $8,1 \%$ & 37 & $0,3 \%$ & 3 & $0,6 \%$ \\
\hline 27 & Investment Management & $8,0 \%$ & 100 & $0,7 \%$ & 8 & $1,5 \%$ \\
\hline 28 & Office/Retail Demand & $7,8 \%$ & 90 & $0,6 \%$ & 7 & $1,3 \%$ \\
\hline 29 & Research & $7,2 \%$ & 139 & $1,0 \%$ & 10 & $1,9 \%$ \\
\hline 30 & Public RE Mngmt & $7,1 \%$ & 28 & $0,2 \%$ & 2 & $0,4 \%$ \\
\hline 30 & PPDV Cycles Investor/User & $7,1 \%$ & 14 & $0,1 \%$ & 1 & $0,2 \%$ \\
\hline 32 & Workplace/Office & $6,8 \%$ & 73 & $0,5 \%$ & 5 & $1,0 \%$ \\
\hline 32 & Miscellaneous Investment Topics & $6,8 \%$ & 323 & $2,3 \%$ & 22 & $4,2 \%$ \\
\hline 34 & Stock, IPOs & $6,5 \%$ & 138 & $1,0 \%$ & 9 & $1,7 \%$ \\
\hline 35 & CRE Holdings/Securitization & $6,3 \%$ & 16 & $0,1 \%$ & 1 & $0,2 \%$ \\
\hline 36 & $\begin{array}{l}\text { Green Building Assesment/ } \\
\text { Certificates }\end{array}$ & $5,6 \%$ & 36 & $0,3 \%$ & 2 & $0,4 \%$ \\
\hline 37 & Energy Consumption & $5,6 \%$ & 18 & $0,1 \%$ & 1 & $0,2 \%$ \\
\hline 38 & ROI & $5,3 \%$ & 132 & $0,9 \%$ & 7 & $1,3 \%$ \\
\hline 39 & CRE, general & $5,2 \%$ & 58 & $0,4 \%$ & 3 & $0,6 \%$ \\
\hline 40 & Land Development & $5,0 \%$ & 119 & $0,8 \%$ & 6 & $1,1 \%$ \\
\hline
\end{tabular}


Tab. 11 Impact Factor deutscher Konferenzbeiträge

\begin{tabular}{|c|c|c|c|c|c|}
\hline $\begin{array}{l}\text { Rg. Impact } \\
\text { Factor D }\end{array}$ & Themenbereich & Anteil D an Global & $\begin{array}{l}\text { Anteil Thema } \\
\text { an Global }\end{array}$ & $\begin{array}{l}\text { Impact } \\
\text { Factor D }\end{array}$ & $\begin{array}{l}\text { Rg. } \\
\text { Global }\end{array}$ \\
\hline 1 & Education & $8,7 \%$ & $2,4 \%$ & 20,53 & 9 \\
\hline 2 & REITs & $3,8 \%$ & $5,2 \%$ & 19,82 & 2 \\
\hline 3 & $\begin{array}{l}\text { Valuation (Models/ } \\
\text { Methods) }\end{array}$ & $3,6 \%$ & $4,8 \%$ & 16,99 & 3 \\
\hline 4 & $\begin{array}{l}\text { Retail/Office/Logistic Real } \\
\text { Estate Markets }\end{array}$ & $4,2 \%$ & $3,7 \%$ & 15,58 & 4 \\
\hline 5 & $\begin{array}{l}\text { Miscellaneous Investment } \\
\text { Topics }\end{array}$ & $6,8 \%$ & $2,3 \%$ & 15,58 & 10 \\
\hline 6 & $\begin{array}{l}\text { Housing Market (incl. } \\
\text { Bubbles, Dynamics) }\end{array}$ & $2,1 \%$ & $7,1 \%$ & 14,87 & 1 \\
\hline 7 & $\begin{array}{l}\text { Inter-/National Real Estate } \\
\text { Markets }\end{array}$ & $4,9 \%$ & $3,0 \%$ & 14,87 & 5 \\
\hline 8 & Funds & $15,4 \%$ & $0,9 \%$ & 14,16 & 33 \\
\hline 9 & Portfolio Management & $9,6 \%$ & $1,3 \%$ & 12,04 & 19 \\
\hline 10 & Securities & $8,3 \%$ & $1,4 \%$ & 11,33 & 18 \\
\hline 11 & Risk Management & $11,9 \%$ & $0,9 \%$ & 11,33 & 31 \\
\hline 12 & Public Private Partnership & $22,6 \%$ & $0,4 \%$ & 8,50 & 71 \\
\hline 13 & RE Rental Demand Cycles & $40,7 \%$ & $0,2 \%$ & 7,79 & 95 \\
\hline 14 & Property Development & $8,1 \%$ & $0,9 \%$ & 7,08 & 36 \\
\hline 15 & Research & $7,2 \%$ & $1,0 \%$ & 7,08 & 28 \\
\hline 16 & Housing Investment & $4,1 \%$ & $1,6 \%$ & 6,37 & 16 \\
\hline 17 & Stock, IPOs & $6,5 \%$ & $1,0 \%$ & 6,37 & 29 \\
\hline 18 & CREM (narrow definition) & $11,5 \%$ & $0,6 \%$ & 6,37 & 57 \\
\hline 19 & Sustainable Housing & $13,4 \%$ & $0,5 \%$ & 6,37 & 65 \\
\hline 20 & Investment Management & $8,0 \%$ & $0,7 \%$ & 5,66 & 47 \\
\hline 21 & $\begin{array}{l}\text { RE Company/Industry } \\
\text { Strategy }\end{array}$ & $8,8 \%$ & $0,6 \%$ & 5,66 & 50 \\
\hline 22 & Real Estate Services & $9,0 \%$ & $0,6 \%$ & 5,66 & 52 \\
\hline 23 & Appraisal & $2,9 \%$ & $1,7 \%$ & 4,96 & 13 \\
\hline 24 & Homeownership/HOS & $3,0 \%$ & $1,7 \%$ & 4,96 & 15 \\
\hline 25 & ROI & $5,3 \%$ & $0,9 \%$ & 4,96 & 32 \\
\hline
\end{tabular}

ermittelten Organisation zugeordnet worden. ${ }^{19}$ Insgesamt wurden 80 Institutionen identifiziert, an denen die Beiträge erstellt wurden. Um unter die nachfolgend dargestellten Top- $25 \mathrm{zu}$ gelangen (die zusammen $469=88 \%$ der Beiträge deutscher Autoren darstellen), reichten 3 Beiträge aus. In Anbetracht der Sensibilität und Missinterpretationsfähigkeit sei explizit darauf hingewiesen, dass aus diesen Daten

- keine Aussagen zur Qualität der Institutionen abgeleitet werden können, da jeder Beitrag unabhängig von seiner Qualität gleich stark gewichtet wurde,

\footnotetext{
${ }^{19} \mathrm{Da}$ bei den ERES-Konferenzen die jeweilige Institution der Autoren nicht veröffentlicht wird, wurde diese entweder auf Basis anderweitig vorliegender Informationen (z. B. Beiträgen desselben Autors auf anderen Konferenzen) oder über Internetrecherche (dann zum Zeitpunkt der Recherche, d. h. Ende 2013) manuell ermittelt.
} 
- keine Aussagen zur Produktivität von Autoren abgeleitet werden können, da erstens diese ihre Institutionen gewechselt oder in Co-Autorenschaft an zahlreichen weiteren Beiträgen maßgeblichen Anteil gehabt haben können,

- ebenso keine Aussagen zur Produktivität von Autoren abgeleitet werden können, da zweitens die an den Institutionen zur Verfügung stehenden Ressourcen nicht berücksichtigt wurden,

- kein zeitadjustiertes Bild gezeichnet wird, da die Institutionen über sehr unterschiedlich lange Zeiträume mit einer schwankenden Anzahl an Personen am Konferenzgeschehen teilgenommen haben.

Um jedes Missverständnis zu vermeiden, haben wir deshalb in diesem Papier explizit auf das Herunterbrechen der Anzahl an Beiträgen auf einzelne Institutionen verzichtet, obwohl diese Zahlen den Autoren vorliegen. Vielmehr soll die nachfolgende Liste einen ersten Eindruck davon vermitteln, an welchen Institutionen und vor allem in welchen Sektoren der Forschungslandkarte immobilienwirtschaftliche Forschung in Deutschland betrieben wird (Tab. 12).

Betrachtet man die Gesamtzahl der Beiträge, ist wenig überraschend, dass die Forschungsbeiträge der untersuchten Konferenzen vor allem von Universitäten kommen (im Schnitt der letzten 20 Jahre zu 72\%). Der Anteil der Beiträge von Fachhochschulen und Forschungszentren betrug im selben Zeitraum 10\% respektive 4\%. Wissenschaftliche Einrichtungen trugen somit zu $86 \%$ zu den deutschen Konferenzbeiträgen bei, Unternehmen, Verbände und Sonstige zu 14\%. Darüber hinaus wird aus der Tabelle eine zeitliche Dynamik deutlich. Zum einen zeigt sich ein deutliches Wachstum von Beiträgen aus Deutschland: Die Anzahl stieg von 36 Beiträgen zwischen 1993-1997 und 54 zwischen 1998-2002 auf 187 zwischen 2003-2007 und weiter auf 256 zwischen 2008-2012. Das entspricht einer durchschnittlichen Wachstumsrate von 10,3\% pro Jahr. Während in der ersten 5-Jahres-Scheibe (von 1993-1997) der Anteil von Unternehmensbeiträgen noch bei 33\% lag, betrug er (bei fast gleicher absoluter Anzahl) zwischen 2008-2012 nur noch 4\%. „Gewinner“ dieser Entwicklung waren insbesondere Universitäten, in kleinerem Maßstab aber auch Forschungszentren und Fachhochschulen, also die wissenschaftlichen Einrichtungen, wohingegen der Anteil der Beiträge von Praktikern zurückging.

\section{Ergebnisse und Limitationen des Forschungsansatzes}

Immobilienwirtschaftliche Forschung ist - im Vergleich zu vielen anderen Feldern wirtschaftswissenschaftlicher Forschung - noch sehr jung, dabei aber inhaltlich angesichts des Forschungsobjekts Immobilie extrem vielfältig. Trotz der rasanten Entwicklung der Forschungsaktivitäten gibt es jedoch erst vergleichsweise wenige wissenschaftliche Publikationsorgane, die in Bezug auf die Erhebung des Forschungsstandes der Forschungsgemeinschaft systematisch ausgewertet werden könnten. Insofern basiert die vorliegende Forschung auf der manuellen Sammlung, Eingabe und Codierung von Beiträgen internationaler immobilienwirtschaftlicher Konferenzen. Eine Metastudie dieser Art stellt einen guten Überblick dar, birgt aber immer das Risiko von Lücken sowie von im Detail diskutierbaren Zuordnungen, was 
Tab. 12 Deutsche Forschungsinstitutionen mit mehr als 3 Beiträgen. (nach Organisationstyp, alphabetisch; Anzahl und Anteil der Beiträge stellen Gesamtoutput aller 80 Institutionen dar)

\begin{tabular}{llllll}
\hline Organisationstyp und & 1993-2012 & 1993-1997 & 1998-2002 & 2003-2007 & 2012
\end{tabular}

Institutsname

Fachhochschulen

Forschungslandschaft

8 Institutionen, davon mit mehr als drei Konferenzbeiträgen: HAWK

Hildesheim/Holzminden, HfWU Hochschule für Wirtschaft und Umwelt Nürtingen-Geislingen

$\begin{array}{llllll}\text { Anzahl der Beiträge } & 54 & 2 & 2 & 22 & 28 \\ \text { In \% von GESAMT } & 10 \% & 6 \% & 4 \% & 12 \% & 11 \%\end{array}$

Forschungszentren

Forschungslandschaft

8 Institutionen, davon mit mehr als drei Konferenzbeiträgen: idw Institut der deutschen Wirtschaft Köln, ZEW Manheim/Centre for European Economic Research

\section{Anzahl der Beiträge \\ In \% von GESAMT \\ Universitäten}

Forschungslandschaft

\begin{abstract}
Anzahl der Beiträge
In \% von GESAMT

Unternehmen
\end{abstract}

Forschungslandschaft

\begin{abstract}
Anzahl der Beiträge
In \% von GESAMT

Sonstige
\end{abstract}

Forschungslandschaft

Anzahl der Beiträge

In \% von GESAMT

GESAMT (in \%)

$\begin{array}{lllll}22 & 0 & 1 & 3 & 18 \\ 4 \% & 0 \% & 2 \% & 2 \% & 7 \%\end{array}$

24 Institutionen, davon mit mehr als drei Konferenzbeiträgen: BauhausUniversität Weimar, European Business School (EBS)/REMI, FU Berlin, Johann-Wolfgang Goethe-Uni Frankfurt, KIT Karlsruhe Institute of Technology/Uni Karlsruhe, RWTH Aachen, TU Berlin, TU Darmstadt, TU Dortmund, TU Kaiserslautern, Uni Hamburg, Uni Leipzig, Uni Mannheim, Un i Stuttgart, Uni Regensburg/IREBS

$\begin{array}{lllll}382 & 20 & 41 & 123 & 198 \\ 72 \% & 56 \% & 76 \% & 66 \% & 77 \%\end{array}$

37 Institutionen, davon mit mehr als drei Konferenzbeiträgen: Credit Suisse Asset Management, Hypo Vereinsbank AG, IPD Investment Property Databank GmbH/DID, MEAG MUNICH ERGO AssetManagement GmbH, IVG Immobilien AG, WLS ENGINEERING+PROJECTMANAGEMNT $\mathrm{GmbH}$

$\begin{array}{lllll}67 & 12 & 9 & 35 & 11 \\ 13 \% & 33 \% & 17 \% & 18 \% & 4 \%\end{array}$

3 Verbände und Privatpersonen

$\begin{array}{lllll}8 & 2 & 1 & 4 & 1 \\ 1 \% & 5 \% & 1 \% & 2 \% & >1 \% \\ \mathbf{5 3 3 ( 1 0 0 \% )} & \mathbf{3 6 ( 7 \% )} & \mathbf{5 4}(\mathbf{1 0} \%) & \mathbf{1 8 7 ( 3 5 \% )} & \mathbf{2 5 6}\end{array}$

bei der Verwendung der hier dargestellten Ergebnisse und Schlussfolgerungen für nachfolgende Entscheidungen berücksichtigt werden sollte.

Nicht Ziel dieses Papers ist es, den Beitrag spezifischer Forschungsschwerpunkte zur Zielerreichung der jeweiligen Lehrstühle oder Forschungsinstitute zu beurteilen, oder die Chancen oder Risiken einzelner Themengebiete für die Zukunft vorauszusagen. Allerdings ermöglicht die „Bilanzierung“ von Forschungsbeiträgen es den Forschern, diese Bewertung im Abgleich mit eigenen Interessen, Stärken und Schwächen sowie ihren Rahmenbedingungen selbst vorzunehmen. Im Vergleich zur internationalen Forschung kann es insofern auch durchaus gerechtfertigt sein, wenn sich die Forschungsagenden deutscher Wissenschaftler vom internationalen Durchschnitt signifikant unterscheiden sollten, weil bewusst auf Nischenstrategien 
gesetzt oder sich den hierzulande besonders relevanten Themenstellungen, die von regional begrenzter Problematik sind, zugewendet wird, und hierzulande relevante, spezifische rechtliche, ökologische, soziologische oder ökonomische Fragestellungen behandelt werden. Während sich anhand der gezeigten Analysen somit zwar Aussagen darüber treffen lassen, ob die Forschung der Autoren im Mainstream lag, müsste eine Bewertung der Relevanz der Forschungsbeiträge vor dem Hintergrund der individuellen Rahmenbedingungen von Forschern und Institutionen in Deutschland in weiteren Analysen mit noch zu entwickelnden operationalisierbaren Kriterien erst noch erfolgen.

Summa summarum zeigen die hier unternommenen Analysen die inhaltliche und quantitative Entwicklung der immobilienwirtschaftlichen Forschung in Deutschland in der Gesamtheit auf und geben einen strukturierten Überblick über die in Deutschland erforschten Themenfelder sowie die hieran beteiligten Institutionen, ohne allerdings Aussagen zu Rigor oder Relevanz der Beiträge Einzelner zu treffen.

Open Access Dieser Artikel wird unter der Creative Commons Namensnennung 4.0 International Lizenz (http://creativecommons.org/licenses/by/4.0/deed.de) veröffentlicht, welche die uneingeschränkte Nutzung, Verbreitung und Wiedergabe für beliebige Zwecke erlaubt, sofern Sie den/die ursprünglichen Autor(en) und die Quelle ordnungsgemäß nennen, einen Link zur Creative Commons Lizenz beifügen und angeben, ob Änderungen vorgenommen wurden.

\section{Literatur}

Hardin, W. G. III, Liano, K., \& Chan, K. C. (Influential, 2006). Influential journals, institutions and researchers in real estate. Real Estate Economics, 34(3), 457-478.

Hardin, W. G. III, Liano, K., \& Chan, K. C. (Citation, 2007). Citation proportions evaluation of real estate research. Journal of Real Estate Research, 15(3), 383-396.

Johnson, L. L., Roulac, S. E., \& Followill, R. A. (ARES Paper, 1996). American Real Estate Society Annual Meeting Paper Presentations: The First Decade (1985-94). Journal of Real Estate Research, 12(2), 195-208.

Kämpf-Dern, A., \& Pfnür, A. (Grundkonzept, 2009). Grundkonzept des Immobilienmanagements. Ein Vorschlag zur Strukturierung immobilienwirtschaftlicher Managementaufgaben. In: A. Pfnür (Hrsg.), Arbeitspapiere zur immobilienwirtschaftlichen Forschung und Praxis (Bd. 14).

Kämpf-Dern, A., Pfnür, A., \& Roulac, S. E. (Perspectives, 2013). Real Estate Perspectives as Major Cluster Attributes for the Analysis of the Last Decades' Real Estate Research. ARES Conference Paper, Spring 2013, Kailua Kona, Hawaii.

Kuhn, T. S. (Scientific Revolutions, 2012). The structure of scientific revolutions, 1996 (4. Aufl.). Chicago: University of Chicago Press.

Mayring, P. (Inhaltsanalyse 2010). Qualitative Inhaltsanalyse: Grundlagen und Techniken (11. Aufl.). Weinheim: Beltz Verlag.

Miles, M. B., \& Huberman, M. (Qualitative, 1994). Qualitative data analysis. Los Angeles: SAGE Publications.

Pfnür, A. (Immobilienmanagement, 2011). Modernes Immobilienmanagement (3. Aufl.). Berlin: Springer Verlag.

Roulac, S. E., Dotzour, M. O., Cheng, P., \& Webb, J. R. (Evolving, 2005). Evolving research priorities: the contents of land economics. Land Economics, 81(4), 457-476.

Schrader, U. u., \& Hennig-Thurau, T. (VHB-JOURQUAL, 2009). VHB-JOURQUAL2: Method, results, and implications of the German academic association for business research's journal ranking. Business Research Journal, 2(2), 180-204.

Schulte, K.-W. (2014): Immobilienökonomie als Wissenschaftsdisziplin - was braucht Europa? In: Gesellschaft für Immobilienwirtschaftliche Forschung e. V. (Hrsg.), Gif im Fokus H. 1/2014, S. 5-6. 
Schulte, K.-W., Bulwien, H., Sturm, V. u., \& Zimmermann, M. (Forschungsschwerpunkte, 2004). Forschungsschwerpunkte der Immobilienökonomie. Ergebnisse empirischer Studien. Zeitschrift für Immobilienökonomie, 1(2004), 6-26.

Strauss, A., \& Corbin, J. (Qualitative, 2008): Basics of Qualitative Research Techniques and Procedures for Developing Grounded Theory (3. Aufl.). Los Angeles: SAGE Publications.

Winson-Geideman, K., \& Evangelopoulos, N. (Topics, 2013). Topics in real estate research, 1973-2010: A latent semantic analysis. Journal of Real Estate Literature, 21(1), 59-76.

Wittgenstein, L. (Philosophical Investitigations, 1953). Philosophical Investigations (PI). In G. E. M. Anscombe \& R. Rhees (Hrsg.), G.E.M. Anscombe (trans.). Oxford: Blackwell. 